\title{
Modulating Electronic Properties of Dinitrosoarene Polymers
}

\author{
Lujo Matasović, ${ }^{\mathrm{a}, \mathrm{b}}$ Barbara Panić, ${ }^{\mathrm{a}}$ Matej Bubaš, ${ }^{\mathrm{c}}$ Hrvoj Vančik, ${ }^{\mathrm{a}}$ Ivana Biljan, ${ }^{\text {a }}$ Igor \\ Rončević*b,d \\ ${ }^{a}$ Department of Chemistry, Faculty of Science, University of Zagreb, Horvatovac 102A, 10000 Zagreb, Croatia \\ ${ }^{b}$ Institute of Organic Chemistry and Biochemistry of the CAS, Flemingovo nám. 2, 16610 Prague 6, Czech Republic \\ ${ }^{c}$ Ruđer Bošković Institute, Bijenička cesta 54, 1000 Zagreb, Croatia \\ ${ }^{d}$ Department of Chemistry, University of Colorado, Boulder, Colorado 80309-0215, United States \\ email: ibiljan@chem.pmf.hr, igor.roncevic@uochb.cas.cz
}

TOC Graphic

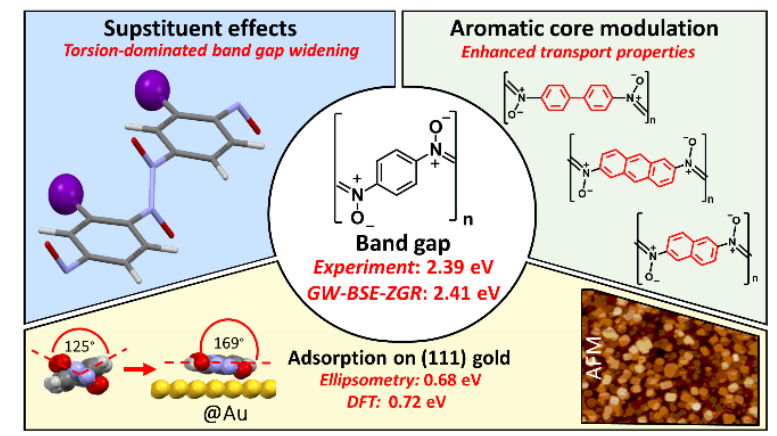

\begin{abstract}
Using the dinitrosobenzene polymer (1) as an example, we explore how the electronic, transport, and optical properties of a conjugated organic semiconductor can be modulated. Combining computational and experimental tools, we explore the effects of solid-state packing, backbone torsion, surface adsorption, the conjugation in the aromatic core, and substituents. The band gap $\left(E_{\mathrm{g}}\right)$ and optical spectrum of $\mathbf{1}$ are calculated using both GW-BSE with zero-gap renormalization (ZGR) and hybrid TD-DFT, with the former method predicting a value $(2.41 \mathrm{eV})$ in excellent agreement with our diffuse reflectance spectroscopy measurements $(2.39 \mathrm{eV})$. Using GW-BSE-ZGR, changes occurring upon solid-state packing are separated into a contribution arising from (i) the change in the torsional angle and (ii) the change in the screened Coulombic interaction, which strongly affects the exciton binding energies. Comprehensive hybrid TD-DFT calculations find that the effects of substituents on $E_{\mathrm{g}}$ and on transport properties can mostly be explained through changes in the torsional angle $\theta$, and predict a linear dependence between $\theta$ and $E_{\mathrm{g}}$. Extending the conjugation in the aromatic core is found to enhance transport properties and narrow $E_{\mathrm{g}}$, identifying future synthetic targets. Atomic force microscopy and spectroscopic ellipsometry are used to study 1 adsorbed to a (111) gold surface (1@Au), with the latter method showing a significant narrowing of the band gap to $0.68 \mathrm{eV}$, in good agreement with TD-DFT predictions.
\end{abstract}

\section{INTRODUCTION}

Due to their exceptional versatility, organic semiconductors (OSCs) are a very attractive alternative to traditional silicon- and GaAs-based semiconductors. OSC properties can be tuned to enable their use in field-effect transistors (FETs), ${ }^{1-4}$ photovoltaics, ${ }^{5-8}$ sensors, ${ }^{9-11}$ and flexible electronics. ${ }^{12-14}$ However, rational design of OSCs for a particular purpose is still very challenging, requiring a combination of synthesis, analytical techniques, and modelling. ${ }^{15}$ On the modelling side, calculations based on density functional theory (DFT) with periodic boundary conditions are being increasingly used to investigate the optoelectronic properties of OSCs. Recently, hybrid DFT and its time-dependent formulation (TD-DFT) have been employed to study periodic porphyrin nanostructures, ${ }^{16}$ polyacenes, ${ }^{17-19}$ as well as polythiophenes and polypyrroles, ${ }^{20,21}$ exploring the changes in the band gap $\left(E_{\mathrm{g}}\right)$ 
and electronic structure occurring upon structural modifications, and thus identifying interesting systems and potential synthetic targets.

More accurate methods for describing the band structures and optical properties of solids are the GW approximation and the Bethe-Salpeter equation (BSE), respectively. ${ }^{22-24} \mathrm{GW}-\mathrm{BSE}$ introduces dynamic (frequency-dependent) screening of the Coulombic interaction, resulting in a nearly parameter-free method that goes well beyond density functional theory. Due to its computational complexity (typically quartic scaling with system size), the use of GW-BSE has mostly been limited to inorganic systems with small unit cells. ${ }^{25-28}$

In this paper, we present and explore a new class of OSCs based on the dinitroso aromatic moiety, which allows for the formation of 1-D polymers. The simplest such compound is 1,4dinitrosobenzene, which forms a remarkably stable polymer 1, whose crystal structure (shown in Figure 1; labelled $\mathbf{C 1}$ to distinguish it from single-strand 1) we recently reported. ${ }^{29}$ Aromatic nitroso compounds are attractive for several reasons: (i) the azodioxide bond (which connects the monomers) is formed spontaneously, meaning that polymer formation is very straightforward; ${ }^{30,31}$ (ii) in cryogenic conditions, UV irradiation can induce reversible cleavage of the azodioxide bond, providing a possibility for ON-OFF switching, ${ }^{32-34}$ (iii) $\mathbf{C 1}$ has a herringbone-type packing, ${ }^{20}$ which is well-known to promote intermolecular charge transfer, ${ }^{35,36}$ (iv) it has been proposed ${ }^{20,30}$ that the angle $\theta$ between the azodioxide and the phenyl planes (see Figure 1) strongly influences the electronic properties of C1, by modulating the extent of conjugation along the polymer. Finally, in case of monomers with more than two nitroso groups, two- and three-dimensional polymer networks can be formed..$^{31,37}$
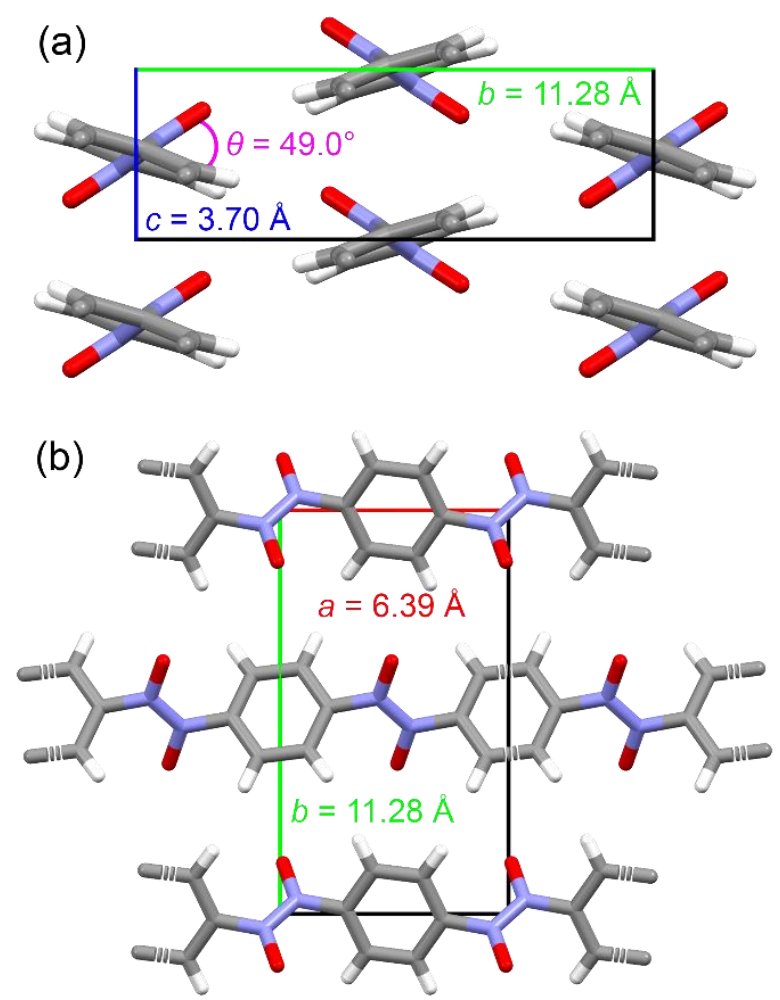

Figure 1. Geometry of $\mathbf{C 1}$, viewed along the $x$ (a) or $z$ (b) direction. The torsional angle $\theta$ and unit cell parameters $a, b$, and $c$ are shown. 
To better understand the factors influencing the band gap of $\mathbf{C 1}$ and related compounds, we recall that band gap engineering typically assumes that the band gap $\left(E_{\mathrm{g}}\right)$ depends on five parameters, which can be written as follows: ${ }^{38,39}$

$$
E_{\mathrm{g}}=E^{3 \mathrm{D}}+E^{\theta}+E^{\mathrm{ASE}}+E^{\mathrm{SUB}}+E^{\mathrm{BLA}}
$$

In equation (1), (i) $E^{3 \mathrm{D}}$ is the solid-state packing of the material; (ii) $E^{\theta}$ is torsion, or the backbone deviation from planarity; (iii) $E^{\mathrm{ASE}}$ is the aromatic stabilization energy; (iv) $E^{\mathrm{SUB}}$ is the presence of substituents; and (v) $E^{\mathrm{BLA}}$ is the bond length alternation. In this paper, we employ a combination of computational and experimental techniques to systematically explore the influence of parameters (i)-(iv) on the band gap of dinitrosoarene polymers (Chart 1). Experimentally, we determine the $E_{\mathrm{g}}$ of $\mathbf{C 1}$ using diffuse reflectance spectroscopy and compare the IR spectra of $\mathbf{1}$ and $\mathbf{C 1}$. Using atomic force microscopy and spectroscopic ellipsometry, we also determine the surface morphology and optical response of thin films of 1 adsorbed to a 111-gold surface (1@Au), respectively. Computationally, we use hybrid TDDFT based on the range-separated HSE06 functional ${ }^{40}$ (TD-HSE) and GW-BSE to evaluate the properties of $\mathbf{1}$ and $\mathbf{C 1}$. The former method is also used to study the effects of torsion, adsorption on a gold surface (1@Au monolayer) and predict the properties of derivatives of $\mathbf{1}$ (Chart 1) differing in their substituents (S2-S9) or aromatic core (A10-A18). These derivatives were chosen as they are amenable to synthesis (e.g., the preparation of $\mathbf{S 8}^{41}$ and $\mathbf{A 1 7 ^ { 4 2 }}$ has been reported). Finally, we evaluate the effect of phonons on $E_{\mathrm{g}}$ by calculating the zero-gap renormalization (ZGR) using an approach recently developed by Kresse et al., ${ }^{43}$ which has, to our knowledge, not yet been applied to organic semiconductors, although the ZGR effects in inorganic systems were found to be significant. ${ }^{34,44} \mathrm{~A}$ similar approach was recently employed in a study by Monserrat et al., where a considerable effect of electronphonon coupling was reported in pentacene molecular crystals. ${ }^{45}$ 

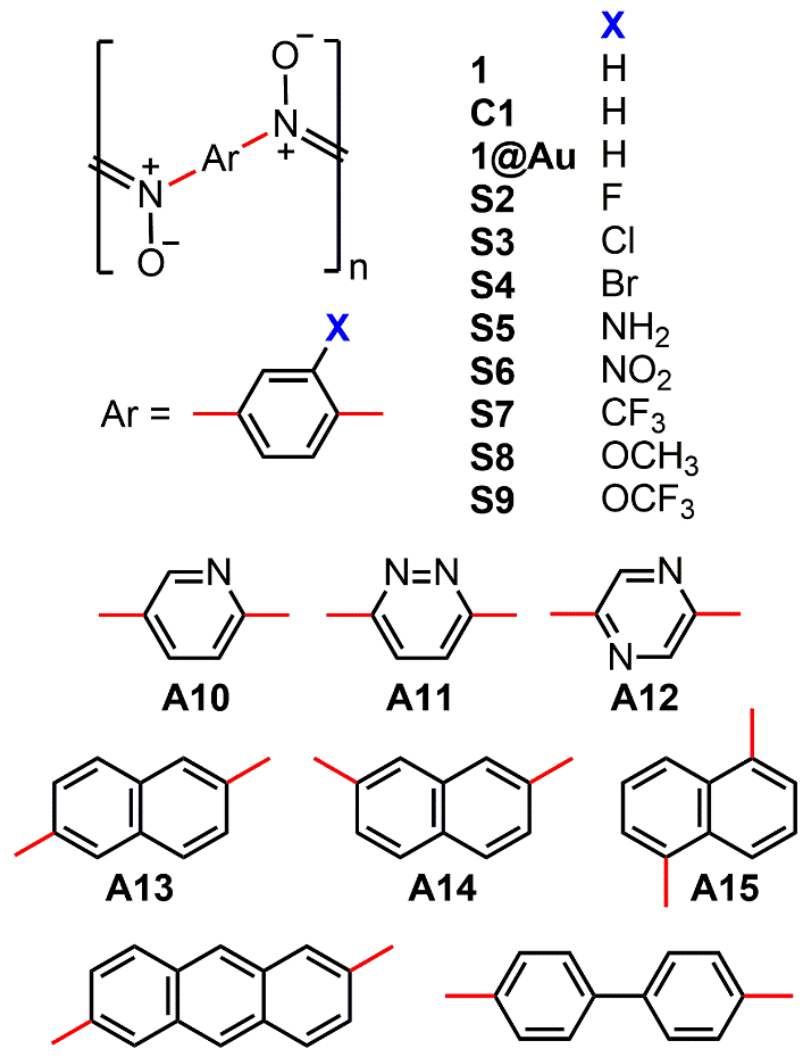

A16

A17<smiles>Cc1ccc2cc3cc4cc(C)ccc4cc3cc2c1</smiles>

A18

Chart 1. Structures of investigated compounds.

The aim of this study is to (i) explore the extent to which the individual parameters influencing $E_{\mathrm{g}}$ can be separated and estimate their contributions, (ii) determine the possible extent of modulation of electronic, transport, and optical properties of $\mathbf{1}$ and identify future synthetic targets, and (iii) compare the predictions made by a rigorous (GW-BSE-ZGR) approach with those obtained by the much more affordable hybrid TD-DFT.

\section{EXPERIMENTAL RESULTS}

(a) Diffuse reflectance spectroscopy. The band gap of $\mathbf{C 1}$ was measured using UV-vis diffuse reflectance spectroscopy. A Tauc plot was used:

$$
\left(F\left(R_{\text {inf }}\right) E\right)^{2}=C_{2}\left(E-E_{\mathrm{g}}\right)
$$

where $F\left(R_{\text {inf }}\right)$ is the Kubelka-Munk reemission function at energy $E$ (details in experimental), and $E_{\mathrm{g}}$ is the band gap. The value of $E_{\mathrm{g}}$ obtained using equation (2) was $2.39 \pm 0.02 \mathrm{eV}$ (Figure 2), confirming $\mathbf{C 1}$ is a wide-gap semiconductor. 


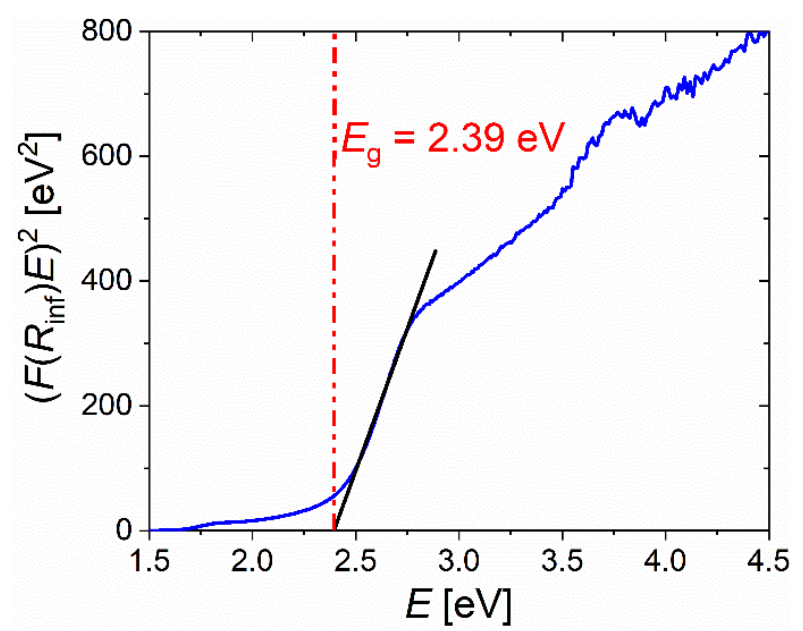

Figure 2. Tauc plot for C1, obtained by UVvis diffuse reflectance spectroscopy. The band gap $E_{\mathrm{g}}$ is denoted by a red line.

(b) Infrared spectroscopy. Infrared spectra of $\mathbf{1}$ and $\mathbf{C 1}$ are nearly identical in the 400-4000 $\mathrm{cm}^{-1}$ range. They are well reproduced by the PBE-revD3BJ ${ }^{46}$ functional (details in experimental), allowing for the complete assignment of IR-active modes (Figure 3 and Table 1). The most prominent signal, corresponding to the asymmetric $E-\mathrm{ON}=\mathrm{NO}$ azodioxide stretching, occurs in the $1200-1300 \mathrm{~cm}^{-1}$ range. Other notable signals are the C-H stretches at $3050-3150 \mathrm{~cm}^{-1}$, in-plane aromatic ring breathing at $\sim 1100 \mathrm{~cm}^{-1}$, and out-of-plane C-H bending at $770-860 \mathrm{~cm}^{-1}$. The modelled spectra overestimate the $\mathrm{C}-\mathrm{H}$ stretching frequencies by about $70-80 \mathrm{~cm}^{-1}$, but otherwise produce an excellent agreement with a mean unsigned error of $11 \mathrm{~cm}^{-1}$ relative to experiment, without any scaling. Signal corresponding to the free nitroso group (around $\left.1520 \mathrm{~cm}^{-1}\right)^{47,48}$ is completely absent, suggesting that the length of polymer strands is very large. 


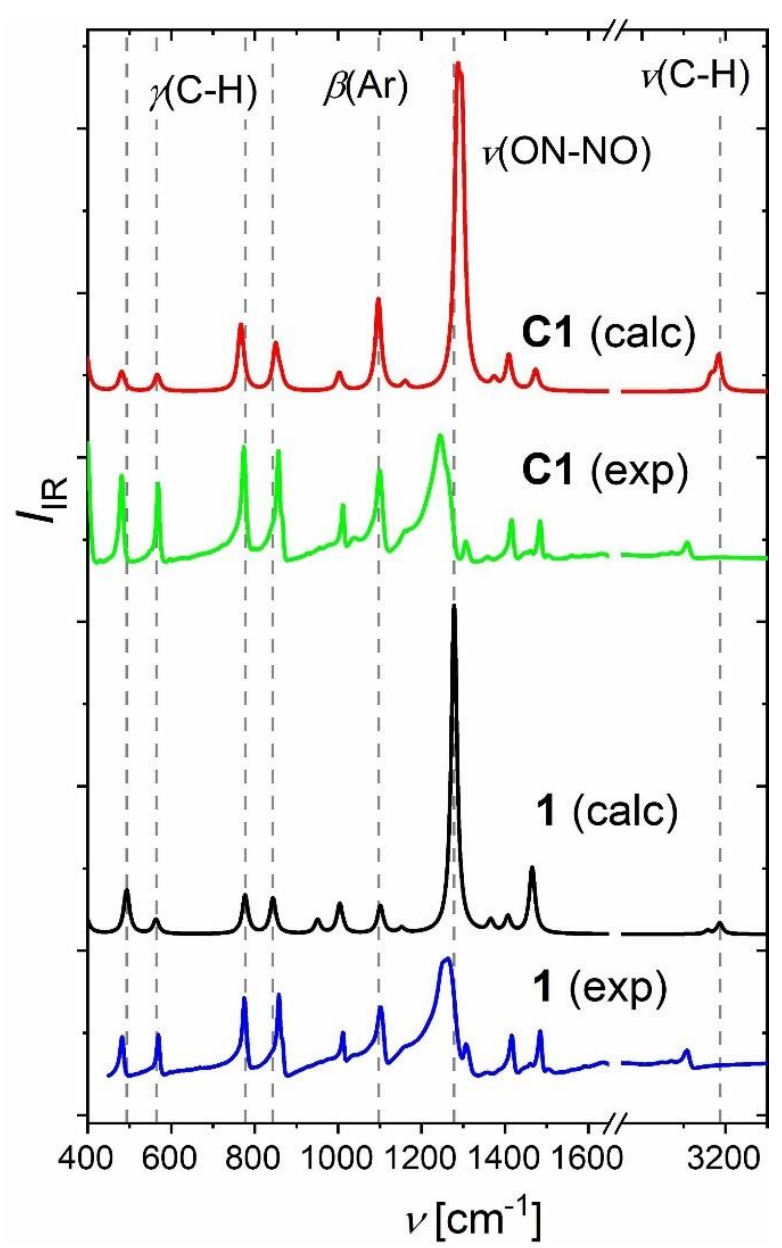

Figure 3. Experimental IR spectra of $\mathbf{1}$ (blue) and C1 (green), along with their DFTcalculated counterparts ( $\mathbf{1}$ black, $\mathbf{C} 1$ red).
Table 1. Experimental $\left(v_{\exp }\right)$ and calculated vibrational $\left(v_{\text {calc }}\right)$ frequencies of $\mathbf{C 1}$ and $\mathbf{1}$ (in $\left.\mathrm{cm}^{-1}\right)$, and their assignments.

\begin{tabular}{|c|c|c|c|c|}
\hline \multirow{2}{*}{ vib. mode ${ }^{a}$} & \multicolumn{2}{|c|}{1} & \multicolumn{2}{|c|}{ C1 } \\
\hline & $v_{\exp }$ & $v_{\text {calc }}$ & $v_{\text {exp }}$ & $v_{\text {calc }}$ \\
\hline$v(\mathrm{C}-\mathrm{H})_{\mathrm{sym}}$ & 3108 & 3187 & 3110 & 3184 \\
\hline$v(\mathrm{C}-\mathrm{H})_{\text {asy }}$ & 3070 & 3157 & 3070 & 3166 \\
\hline$\delta(\mathrm{C}-\mathrm{H})_{\mathrm{sym}}$ & 1485 & 1465 & 1483 & 1474 \\
\hline$\delta(\mathrm{C}-\mathrm{H})_{\text {asy }}$ & 1407 & 1416 & 1415 & 1410 \\
\hline $\begin{array}{l}v(E- \\
\mathrm{ON}=\mathrm{NO})_{\text {asy }}\end{array}$ & 1263 & 1278 & 1248 & 1295 \\
\hline$v(\mathrm{C}-\mathrm{N})_{\text {asy }}$ & 1160 & 1152 & 1159 & 1188 \\
\hline$\rho(\mathrm{C}-\mathrm{H})$ & 1011 & 1004 & 1011 & 1006 \\
\hline$\beta(\mathrm{Ar})$ & 1105 & 1099 & 1101 & 1100 \\
\hline \multirow{4}{*}{$\gamma(\mathrm{C}-\mathrm{H})$} & 857 & 843 & 858 & 850 \\
\hline & 774 & 777 & 772 & 767 \\
\hline & 568 & 565 & 568 & 567 \\
\hline & 482 & 493 & 479 & 481 \\
\hline
\end{tabular}

(c) Atomic Force Microscopy. Comparison of AFM height images of a freshly annealed (111) gold substrate (Figure 4a) and thin films produced by drop-casting solution of monomers of 1 on an $\mathrm{Au}(111)$ surface (1@Au) (Figure 4b) reveals significant changes in surface morphology induced by adsorption of $\mathbf{1}$. The AFM image of $\mathbf{1} @ \mathbf{A u}$ shows close-packed islands, completely covering the (111) gold terraces. The height-profile analysis of AFM data indicates that the heights of islands are mostly in the range from 15 to $30 \mathrm{~nm}$, while their lateral sizes vary from about 50 to $150 \mathrm{~nm}$ (Figure 4c). The RMS roughness value obtained from a $2 \mu \mathrm{m} \times 2 \mu \mathrm{m}$ scan area of $\mathbf{1} @ \mathbf{A u}$ is $66.2 \mathrm{~nm}$. These results suggest that deposition of monomers of $\mathbf{1}$ on a (111) gold surface leads to polymerization and the formation of multilayers which are mostly composed of $\mathbf{1}$.
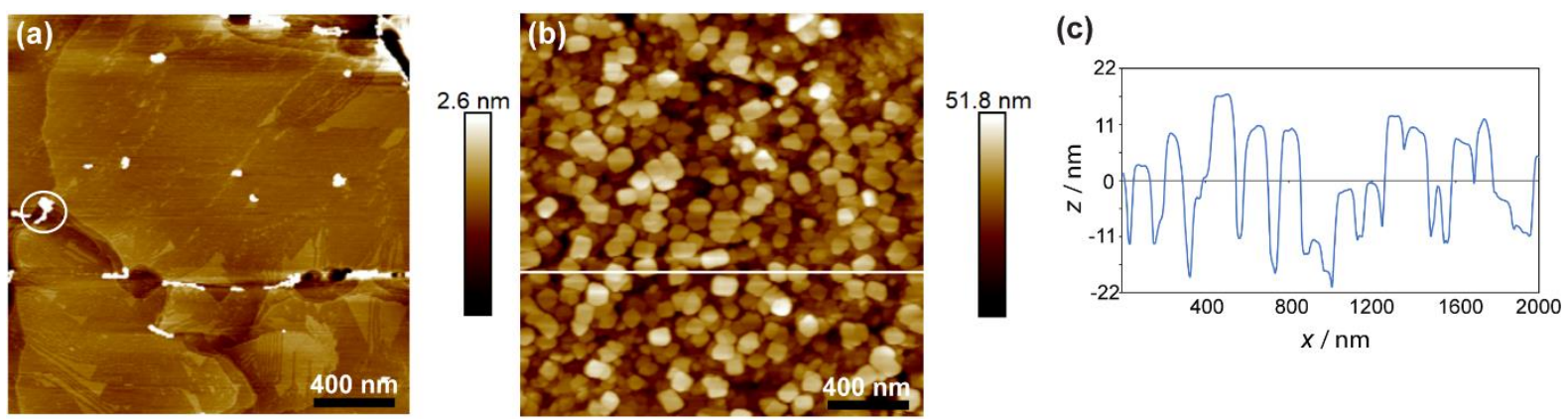
Figure 4. $2 \mu \mathrm{m} \times 2 \mu \mathrm{m}$ AFM height images of (a) bare (111) gold surface, and (b) $\mathbf{1} @ \mathbf{A u}$ prepared by drop-casting solution of monomers of $\mathbf{1}$ on an $\mathrm{Au}(111)$. The corresponding height profile along the white line in (b) is shown in (c). Clusters on the surface of a bare (111) gold substrate (marked with white circle in a) are attributed to environmental contaminants under ambient conditions and surface preparation treatment.

(d) Ellipsometry. The thickness of a thin film of 1 produced by drop-casting on a (111) gold surface $(1 @ \mathbf{A u})$ is $178 \pm 4 \mathrm{~nm}$, as determined by ellipsometry. The imaginary part of the dielectric function displays a peak at $1.03 \mathrm{eV}$ in the IR region, and a broad signal with a maximum around $2.1 \mathrm{eV}$ in the visible part of the spectrum (Figure 5). The band gap, as determined from the Tauc-Lorentz oscillator model, is $0.68 \pm 0.01 \mathrm{eV}$. The index of refraction of $1 @ \mathbf{A u}$ is $\sim 1.5$ in the NIR and visible range, and increases in the UV, which is typical for organic polymers. However, the most notable feature of $\mathbf{1} @ \mathbf{A u}$ is that it exhibits considerable attenuation in the visible and the NIR parts of the spectrum (Figure 5).

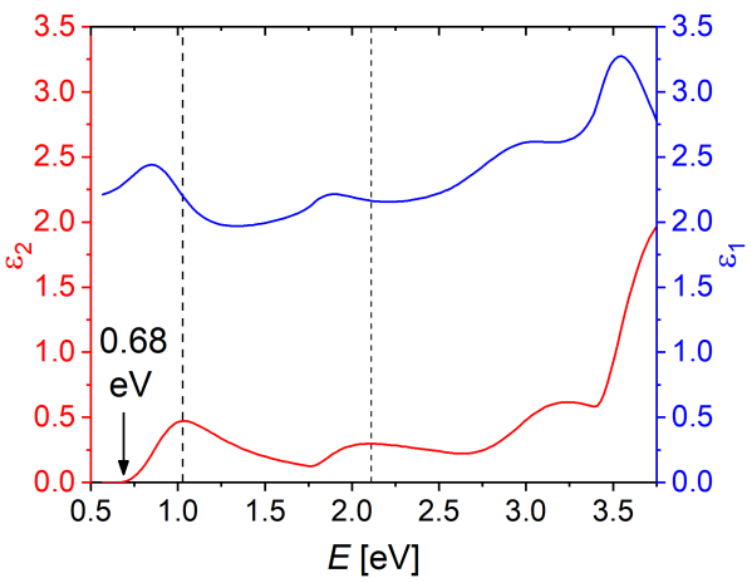

Figure 5. The imaginary (red) and real (blue) parts of the dielectric function of $1 @ \mathbf{A u}$, as determined by spectroscopic ellipsometry. The black arrow denotes the value of $E_{\mathrm{g}}$, while the dashed vertical lines show the positions of absorption maxima.

\section{COMPUTATIONAL RESULTS}

(e) Geometry and phonons. Geometry optimization using the PBE-revD3BJ method yields a geometry and unit cell parameters for $\mathbf{C 1}$ in good agreement with its XRD-determined ${ }^{29}$ structure, with a mean unsigned error of $2.0 \%$ (details in Figure S1 in SI). The same method gives IR spectra in good accord with experiment (cf. section $(b)$ ). Vibrational structures of $\mathbf{C 1}$ and 1 are very similar, with small differences $(<10 \%)$ only at energies below $400 \mathrm{~cm}^{-1}$, as illustrated by a comparison of their phonon band structures (Figure 6; full phonon band structure of $\mathbf{C 1}$ can be found in Figure S5). The ZGR value at $0 \mathrm{~K}$ was determined to be -0.27 $\mathrm{eV}$ for $\mathbf{C 1}$ and $-0.28 \mathrm{eV}$ for $\mathbf{1}$. 


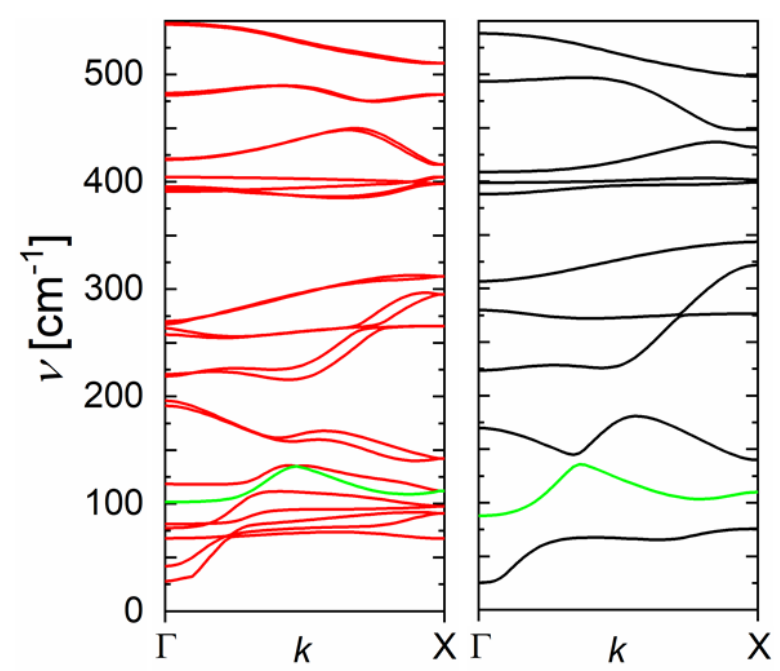

Figure 6. Phonon band structure of 1 (black) and C1 (red) along the polymer chain. Modes corresponding to translation have been omitted. Mode 9 (M9) in C1 and mode 5 (M5) in $\mathbf{1}$ are shown in green. $\mathbf{C 1}$ has two molecules in the unit cell, while $\mathbf{1}$ has only one.

(f) Electronic structure and optical properties. To describe $\mathbf{1}$ and $\mathbf{C 1}$, two approaches were employed. In the first, the electronic structure and transport properties were calculated at the hybrid DFT level using the HSE06 functional, ${ }^{40}$ while its time-dependent variant (TD-HSE) was used for calculations of optical spectra. The second approach (GW-BSE-ZGR) involved a partially self-consistent eigenvalue GW0 calculation ${ }^{49}$ on orbitals obtained using PBE. This was followed by the use of the Bethe-Salpeter equation (BSE) to account for electron-hole interactions and the addition of ZGR corrections to include electron-phonon coupling. These calculations were done on optimized geometries of $\mathbf{1}$ and $\mathbf{C 1}$.

Results are summarized in Table 2 and Figure 7. Relative to experiment, TD-HSE underestimates $E_{\mathrm{g}}$ of $\mathbf{C 1}$ by about $0.23 \mathrm{eV}$, while GW-BSE-ZGR produces excellent agreement with experiment, with an error of only $0.02 \mathrm{eV}$. The exciton binding energies $E_{\mathrm{xb}}$ calculated by TD-DFT and GW-BSE are dramatically different, with hybrid DFT values being much smaller ( $0.01 \mathrm{eV}$ and $0.23 \mathrm{eV}$ for $\mathbf{C 1}$ and 1, respectively) than those obtained using the $a b$ initio approach $(0.53 \mathrm{eV}$ and $1.44 \mathrm{eV})$. However, the effective masses, optical spectra of $\mathbf{C 1}$ (Figure $7 \mathrm{~d}$ ), and the positions of the first strong absorption maximum $E_{\max }$ are quite similar, $3.02 \mathrm{eV}$ for TD-HSE and $3.03 \mathrm{eV}$ for GW-BSE-ZGR, compared to the previously measured ${ }^{48}$ experimental value of $3.10 \mathrm{eV}$. Comparing $\mathbf{C 1}$ and 1, GW-BSE-ZGR predicts they have approximately the same $E_{\mathrm{g}}$ values, while TD-HSE suggests that $E_{\mathrm{g}}$ of $\mathbf{1}$ is smaller by $\sim 0.2 \mathrm{eV}$ relative to $\mathbf{C} \mathbf{1}$.

The band structure of $\mathbf{C 1}$ reveals a direct gap at A and nearly degenerate pairs of conduction and valence bands close to the Fermi level, with flat regions associated with the variation of $k_{\mathrm{y}}$ (G-Y; A-E; C-Y). Relative to HSE, GW opens the gap by $\sim 1.0 \mathrm{eV}$, and otherwise differs from it only along the A-E path, where it produces two distinctly separate bands, while HSE predicts them to be separated in $\mathrm{A}(\pi, 0, \pi)$ and degenerate in $\mathrm{E}(\pi, \pi, \pi)$. The first bright transition is strongly excitonic in nature, and corresponds to the transfer of electron density from the N-N bond and oxygen to the C-N bond (Figure 7e), which may be associated with the cleavage of the azodioxy bond. 
Table 2. Exciton binding energies $\left(E_{\mathrm{xb}}\right)$, band gaps $\left(E_{\mathrm{g}}\right)$, positions of the first strong absorption maximum $\left(E_{\max }\right)$, and effective masses of the valence $\left(m_{\mathrm{e}, \mathrm{VB}}\right)$ and conduction $\left(m_{\mathrm{e}, \mathrm{CB}}\right)$ bands of $\mathbf{1}$ and $\mathbf{C 1}$ (in eV), obtained using the TD-HSE and GW-BSE approaches. Values in parentheses are with the ZGR correction.

\begin{tabular}{|c|c|c|c|}
\hline \multicolumn{2}{|c|}{ Method/cmpd. } & $\mathrm{C1}$ & 1 \\
\hline \multirow{5}{*}{ อ } & $E_{\mathrm{xb}}$ & 0.01 & 0.23 \\
\hline & $E_{\mathrm{g}}$ & 2.16 & 1.97 \\
\hline & $E_{\max }$ & 3.02 & 2.10 \\
\hline & $m_{\mathrm{e}, \mathrm{CB}}$ & 1.42 & 0.64 \\
\hline & $m_{\mathrm{e}, \mathrm{VB}}$ & 0.67 & 0.16 \\
\hline \multirow{5}{*}{ 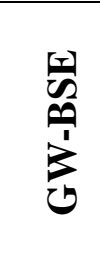 } & $E_{\mathrm{xb}}$ & 0.53 & 1.44 \\
\hline & $E_{\mathrm{g}}\left(E_{\mathrm{g}, \mathrm{ZGR}}\right)$ & $2.67(2.41)$ & 2.67 (2.39) \\
\hline & $E_{\max }\left(E_{\max , \mathrm{ZGR}}\right)$ & $3.30(3.03)$ & 2.67 (2.39) \\
\hline & $m_{\mathrm{e}, \mathrm{CB}}$ & 1.49 & 0.59 \\
\hline & $m_{\mathrm{e}, \mathrm{VB}}$ & 0.61 & 0.12 \\
\hline
\end{tabular}

(a)

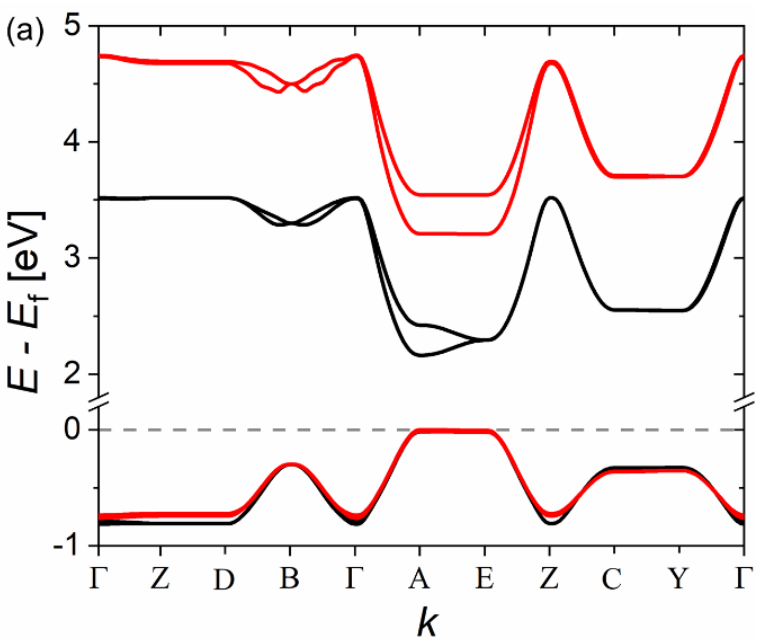

(d)

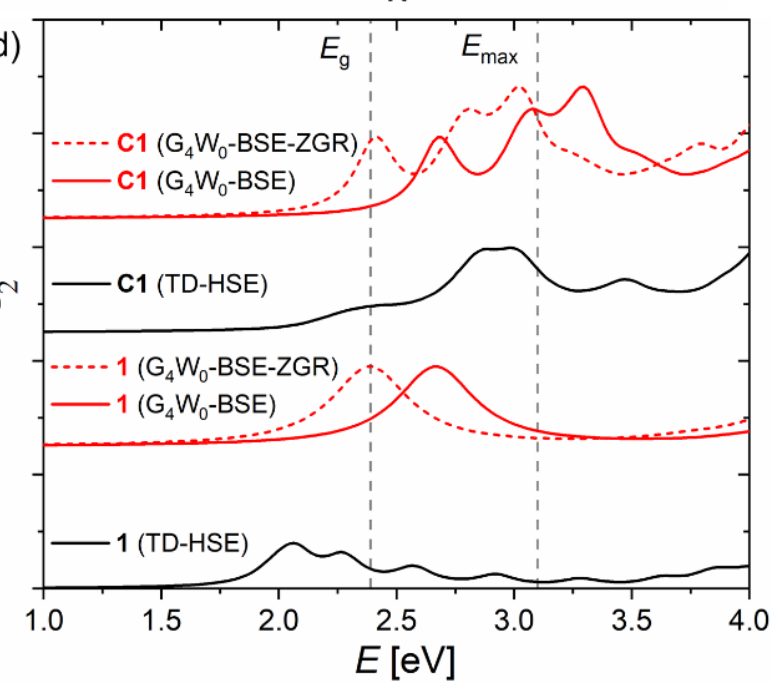

(b)

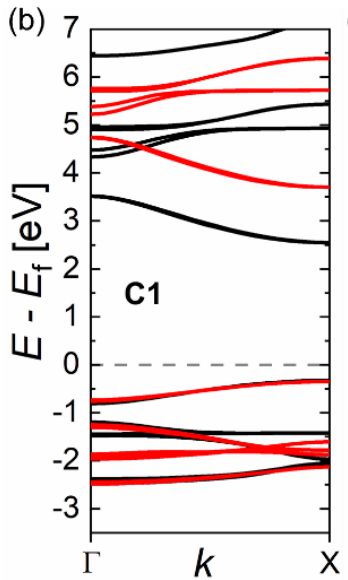

(c) 7

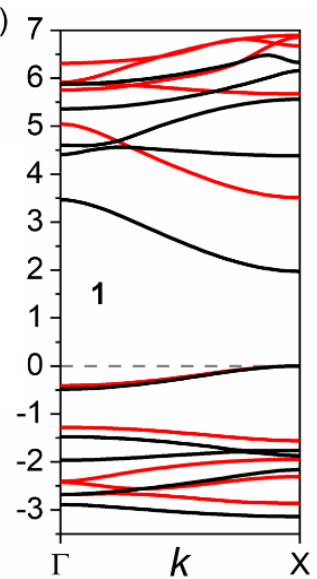

(e)

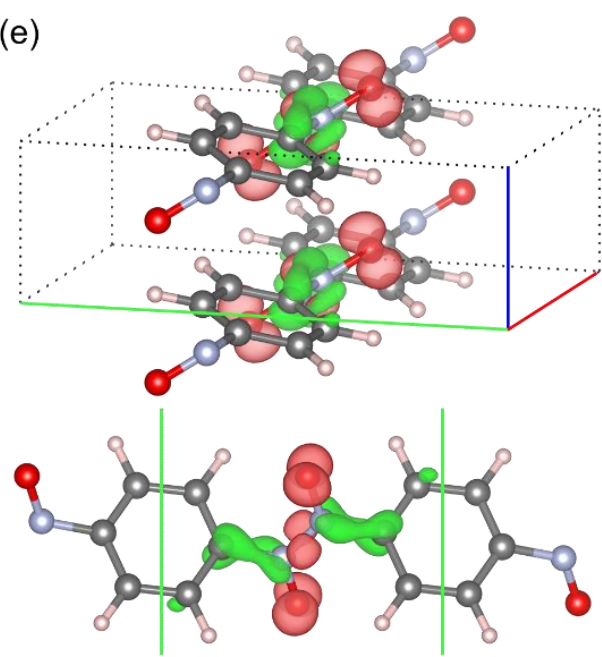

Figure 7. Band structure of $\mathbf{C 1}$ (a) and a comparison of band structures of $\mathbf{C 1}$ (b) and $\mathbf{1}$ (c) along the $\Gamma$-X path, obtained using GW (red) or HSE (black). Fermi level is set to the top of the valence band. (d) Optical spectra of $\mathbf{C 1}$ and 1, obtained using GW-BSE (red, dashed 
offset by ZGR) and TD-HSE (black), with the experimental values of $E_{\mathrm{g}}$ and $E_{\max }$ denoted by vertical lines. (e) Transition density at the A high-symmetry point, associated with $E_{\mathrm{g}}$.

(g) Torsion and packing. Analysis of the optimized geometries of $\mathbf{1}$ and $\mathbf{C 1}$ reveals a significant difference in $\theta$, the angle between the azodioxide and phenyl planes, with the polymer geometries otherwise being virtually identical. In an isolated polymer strand of $\mathbf{1}$ ( $\theta_{1}$ $\left.=37.5^{\circ}\right), \theta$ is about $16.3^{\circ}$ smaller than in $\mathbf{C 1}\left(\theta \mathbf{C 1}=53.8^{\circ}\right)$. To separate the effects of torsion from packing, we performed a GW-BSE calculation of $\mathbf{1}$ with the geometry extracted from

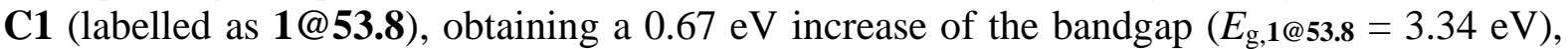
and a $0.28 \mathrm{eV}$ increase in $E_{\mathrm{xb}}\left(E_{\mathrm{xb}, \mathbf{1} @ 53.8}=0.81 \mathrm{eV}\right)$, which gives a very strong dependence $\left(0.041 \mathrm{eV} /{ }^{\circ}\right.$, assuming a linear relationship) of the band gap on $\theta$.

In an alternative approach, we investigated the displacement along phonon modes of $\mathbf{1}$ and C1 which correspond to changes in $\theta$ using TD-HSE. In 1, such a mode (M5) appears at 88 $\mathrm{cm}^{-1}$, while a corresponding mode in $\mathbf{C 1}$ (M9) is at $102 \mathrm{~cm}^{-1}$, with the two showing similar dispersion (Figure 6). The results, shown in Figure 8, show a strong linear dependence of $E_{\mathrm{g}}$ on $\theta$. Linear regression yields $E_{\mathrm{g}}=0.039 \theta \mathrm{eV} /{ }^{\circ}+0.07 \mathrm{eV}\left(R^{2}=0.99982\right)$ for $\mathbf{C 1}$ and $E_{\mathrm{g}}=$ $0.024 \theta \mathrm{eV} /^{\circ}+1.11^{\circ} \mathrm{eV}$ for $\mathbf{1}\left(R^{2}=0.99487\right)$.

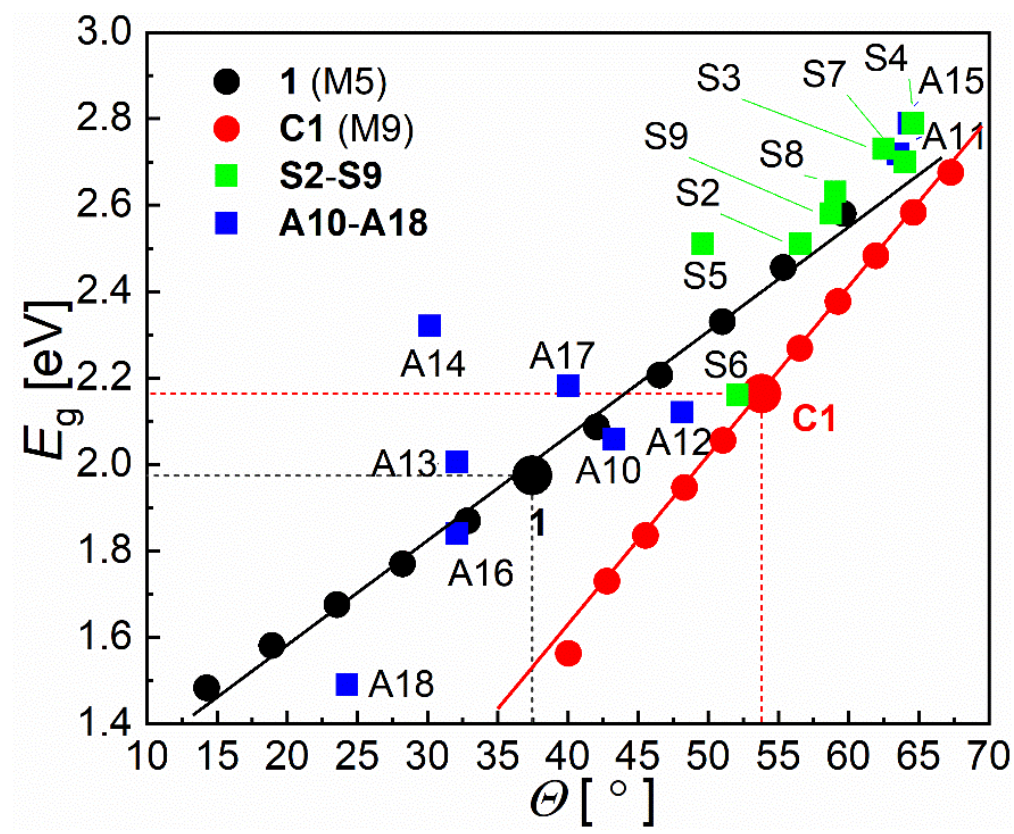

Figure 8. Influence of torsion (displacement along modes M5 and M9 for $\mathbf{1}$ and $\mathbf{C 1}$, respectively) on the TD-HSE bandgap $\left(E_{\mathrm{g}}\right)$ of $\mathbf{1}$ (black dots) and $\mathbf{C 1}$ (red dots). Effect of substituents (green rectangles) and the aromatic core (blue rectangles) is also shown. Equilibrium geometries of $\mathbf{1}$ and C1 are highlighted with dashed lines.

(h) Adsorption of $\mathbf{1}$ on a (111) gold surface. Relaxation of $\mathbf{1}$ on a (111) gold surface (1@ Au monolayer) using the VDW-DF25050 functional results in an adsorption energy of 21.2 $\mathrm{kJ} / \mathrm{mol}$ per dinitrosobenzene unit, and an almost complete planarization $\left(\theta_{1} @ \mathrm{Au}=12.7^{\circ}\right.$, Figure 9) of the polymer. Changes in $E_{\mathrm{g}}$ resulting from adsorption were estimated in two ways: (A) by calculating the band gap difference between $\mathbf{1}$ and $\mathbf{1} @ \mathbf{A u}$ monolayer at the VDW-DF2 level and adding it to the GW-BSE-ZGR value for 1, as well as (B) by TD-HSE calculations of the isolated geometry of $\mathbf{1}$ extracted from $\mathbf{1} @ \mathbf{A u}$ monolayer. Both methods predict a dramatic reduction in the band gap upon adsorption (Table $3 ; E_{\mathrm{g}, \mathrm{A}}=1.09 \mathrm{eV}$; B: $E_{\mathrm{g}, \mathrm{B}}$ 
$=0.75 \mathrm{eV})$, although method $\mathrm{B}$ is in better agreement with the $0.68 \mathrm{eV}$ value obtained by spectroscopic ellipsometry.

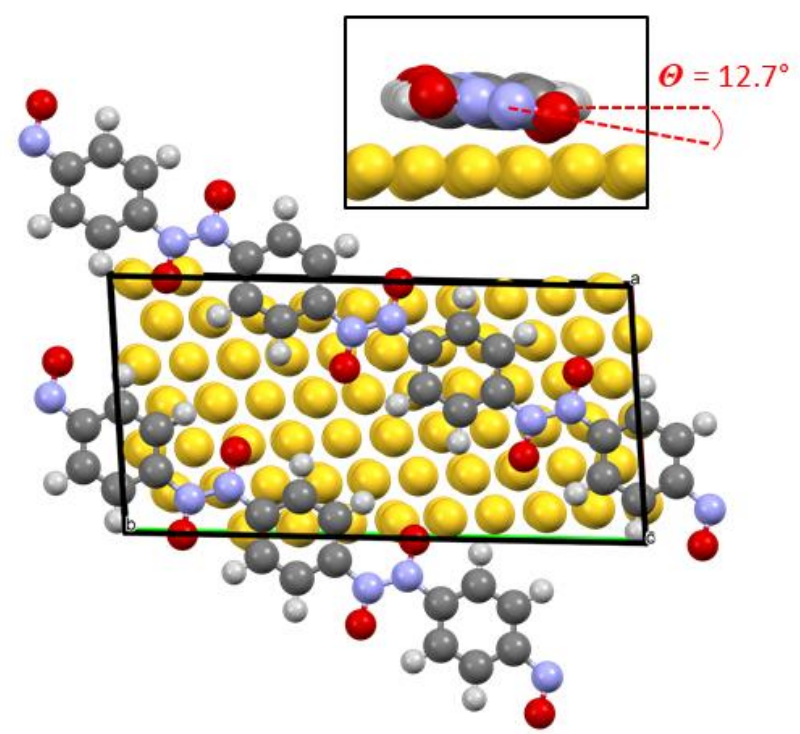

Table 3. Band gap of a monolayer of 1 $(\mathrm{eV})$ after adsorption to the gold surface.

\begin{tabular}{|c|c|}
\hline Method & $E_{\mathrm{g}, 1 @ A u}$ \\
\hline $\begin{aligned} \mathrm{A}: & E_{\mathrm{g}, \mathbf{1}, \mathrm{GW}-\mathrm{BSE}-\mathrm{ZGR}+} \\
& \Delta E_{\mathrm{g}, \mathbf{1} @ \mathbf{A u}-\mathbf{1}, \mathrm{VDW}-\mathrm{DF}}\end{aligned}$ & 1.09 \\
\hline B: $E_{\mathrm{g}, \mathbf{1} @ \mathrm{Au} \text {,isolated,TD-HSE }}$ & 0.75 \\
\hline Ellipsometry & 0.68 \\
\hline
\end{tabular}

Figure 9. Geometry of the 1@Au monolayer along the $\mathrm{z}$ direction, with a cutout highlighting the planarization, and the value of $\theta$.

(i) Substituents. In case of dinitroso dimers, the influence of substituents (see Chart 1) on the band gap is considerable and can be described well using a Hammett equation5151 (Figure S7). The $\rho$ value and correlation coefficient for dimers $\left(0.22 \pm 0.05 \mathrm{eV} ; R^{2}=0.72\right)$ show a significant correlation between $\sigma$ and $E_{\mathrm{g}}$, which is completely absent in polymers $\mathbf{S 2}-\mathbf{S 9}$ ( $\rho=$ $\left.-0.3 \pm 0.2 \mathrm{eV} ; R^{2}=0.003\right)$. Instead, introducing a substituent invariably widens the band gap and decreases dispersion, resulting in larger hole and electron effective masses (Figure 10 and Table 4). Introducing substituents also affects the torsional angle $\theta$, which is shown in Figure 8. 

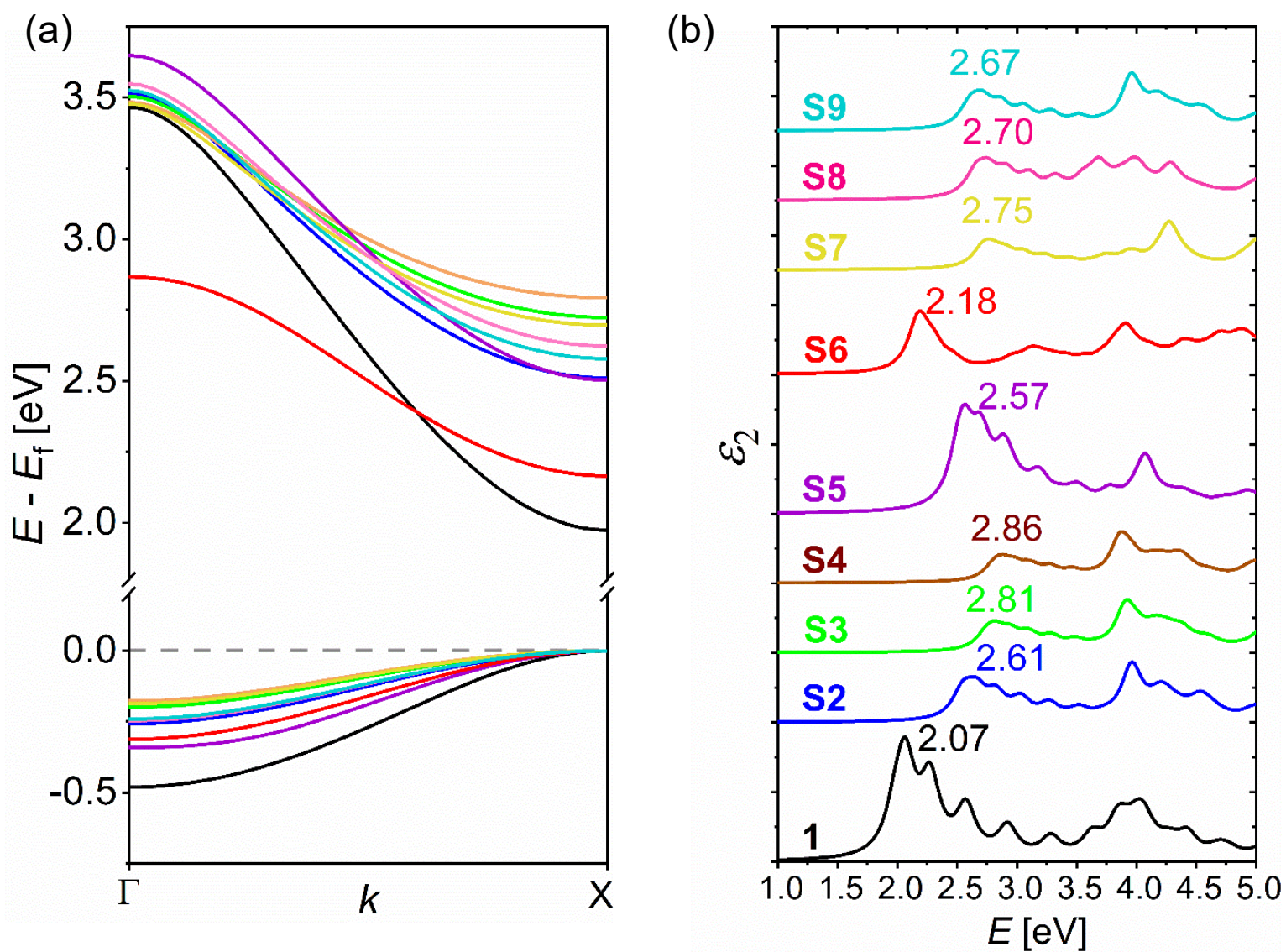

Figure 10. (a) HSE06 valence and conduction bands for $\mathbf{1}$ and its substituted derivatives S2S9. The Fermi level is set at the maximum of the valence band. (b) TD-HSE absorption spectra of $\mathbf{1}$ and $\mathbf{S 2 - S 9 .}$

Table 4. HSE06 Band gaps $\left(\boldsymbol{E}_{\mathrm{g}}\right)$, energies of first absorption maxima $\left(\boldsymbol{E}_{\mathbf{m a x}}\right)$ and effective masses of valence $\left(\boldsymbol{m}_{\mathbf{e}, \mathbf{V B}}\right)$ and conduction bands $\left(\boldsymbol{m}_{\mathbf{e}, \mathbf{C B}}\right)$ for $\mathbf{1}$ and $\mathbf{S 2}-\mathbf{S 9}$.

\begin{tabular}{|l|cccc|}
\hline Cmpd. & $\boldsymbol{E}_{\mathrm{g}}$ & $\boldsymbol{E}_{\mathrm{max}}$ & $\boldsymbol{m}_{\mathrm{e}, \mathrm{VB}}$ & $\boldsymbol{m}_{\mathrm{e}, \mathrm{CB}}$ \\
\hline $\mathbf{1}$ & 1.98 & 2.07 & 0.71 & 0.31 \\
S2 & 2.51 & 2.61 & 1.73 & 0.57 \\
$\mathbf{S 3}$ & 2.73 & 2.81 & 2.49 & 0.82 \\
S4 & 2.79 & 2.86 & 3.03 & 0.99 \\
S5 & 2.51 & 2.57 & 1.08 & 0.43 \\
S6 & 2.16 & 2.18 & 1.32 & 0.61 \\
S7 & 2.70 & 2.75 & 2.68 & 0.83 \\
S8 & 2.63 & 2.70 & 1.87 & 0.65 \\
S9 & 2.58 & 2.67 & 1.90 & 0.62 \\
\hline
\end{tabular}

(j) Influence of the aromatic core. Substituting the phenyl group for a nitrogen-containing heterocycle (A10-A12) widens $E_{\mathrm{g}}$ and diminishes the transport properties (Figure 11 and Table 5). The change is relatively small $\left(\Delta E_{\mathrm{g}} \approx 0.1 \mathrm{eV}\right)$ for pyridine (A10) and pyrazine (A12), but much larger for pyridazine $\left(\mathbf{A 1 1} ; \Delta E_{\mathrm{g}} \approx 0.7 \mathrm{eV}\right.$ ), which also displays a roughly five-fold decrease in the intensity associated with the first absorption maximum relative to $\mathbf{1}$. 
Increasing the aromatic stabilization energy by replacing benzene with naphthalene (A13A15) results in compounds with properties which are highly dependent on the positions of the two nitroso groups. In A13, $E_{\mathrm{g}}$ does not significantly change relative to $\mathbf{1}$, but effective masses are decreased by $30-40 \%$, and the position of the fundamental gap changes from $\mathrm{X}$ to $\Gamma$. On the other hand, in A14 and A15 the conduction and valence bands become strongly localized, which is accompanied by gap widening. Introducing anthracene (A16) narrows $E_{\mathrm{g}}$ to $1.90 \mathrm{eV}$ and strongly decreases the electron $(\sim 40 \%)$ and hole $(\sim 50 \%)$ effective mass. Exchanging phenyl for biphenyl (A17) narrows the gap by about $0.2 \mathrm{eV}$, but increases the absorption intensity as well as the electron and hole mobilities by $20-30 \%$. Finally, the tetracene-based $\mathbf{A 1 8}$ is found to have the lowest gap $\left(E_{\mathrm{g}}=1.68 \mathrm{eV}\right)$ of all the investigated compounds, with transport properties very similar to A16.

(a)

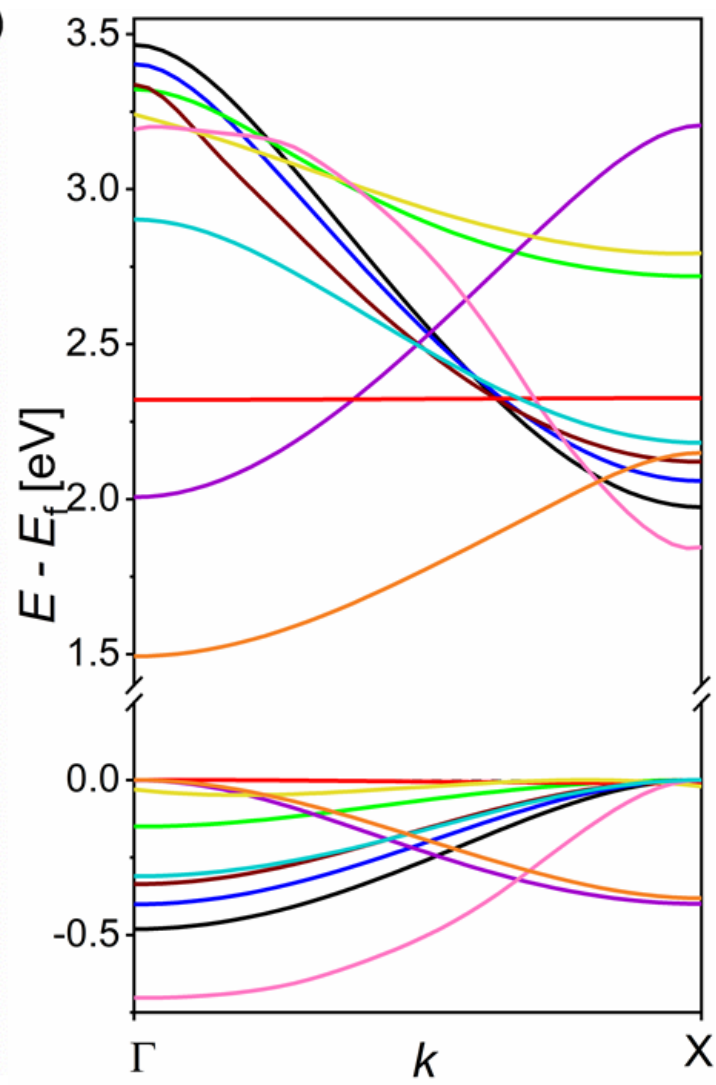

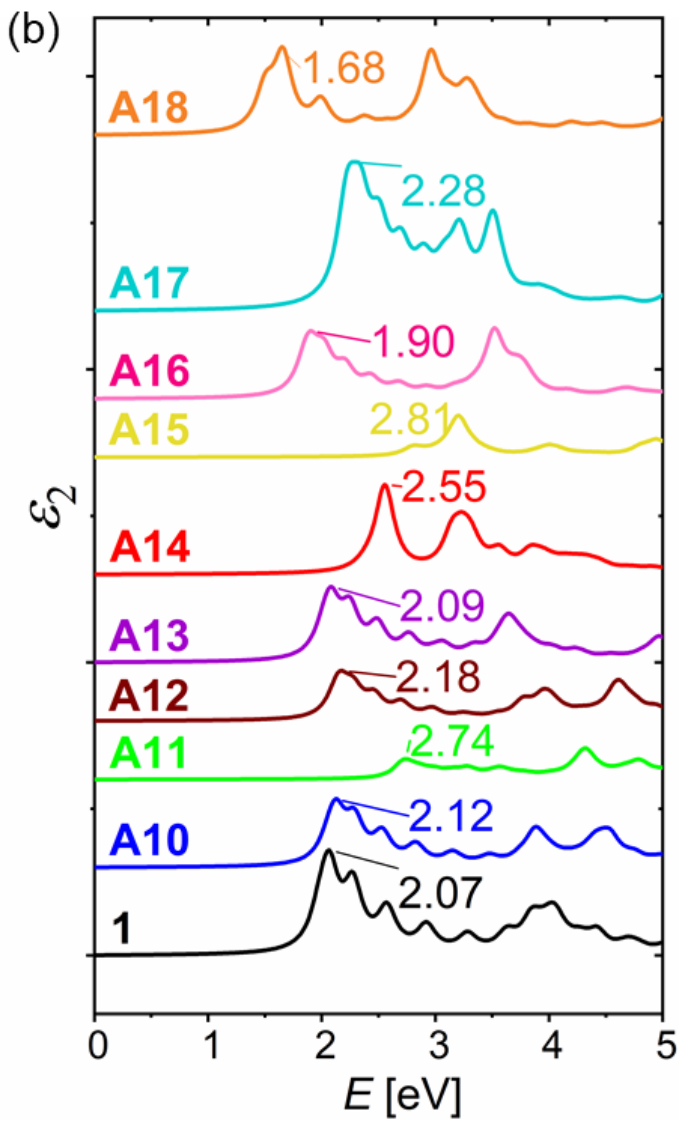

Figure 11. (a) HSE06 valence and conduction bands for 1 and its derivatives A10-A18. The Fermi level is set at the maximum of the valence band. (b) TD-HSE absorption spectra of $\mathbf{1}$ and A10-A18.

Table 5. HSE06 band gaps $\left(\boldsymbol{E}_{\mathrm{g}}\right)$, energies of first absorption maxima $\left(\boldsymbol{E}_{\mathrm{max}}\right)$, effective masses of valence $\left(\boldsymbol{m}_{\mathbf{e}, \mathbf{V B}}\right)$ and conduction bands $\left(\boldsymbol{m}_{\mathbf{e}, \mathbf{C B}}\right)$, and aromatic stabilization energies $\left(\boldsymbol{E}_{\mathbf{A S E}}\right)$ for $\mathbf{1}$ and A10-A18. All energies in $\mathrm{eV}$.

\begin{tabular}{|l|rrrr|r|}
\hline Cmpd. & $\boldsymbol{E}_{\mathbf{g}}$ & $\boldsymbol{E}_{\mathbf{m a x}}$ & $\mathbf{m}_{\mathbf{e}, \mathbf{V B}}$ & $\mathbf{m}_{\mathbf{e}, \mathbf{C B}}$ & $\boldsymbol{E}_{\text {ASE }}$ \\
\hline $\mathbf{1}$ & 1.98 & 2.07 & 0.71 & 0.31 & $1.39^{\mathrm{b}}$ \\
$\mathbf{A 1 0}$ & 2.06 & 2.13 & 0.97 & 0.40 & $1.34^{\mathrm{c}}$ \\
$\mathbf{A 1 1}$ & 2.71 & 2.74 & 4.81 & 1.19 & $1.42^{\mathrm{c}}$ \\
$\mathbf{A 1 2}$ & 2.12 & 2.18 & 1.32 & 0.55 & $1.29^{\mathrm{c}}$
\end{tabular}




\begin{tabular}{|l|lllr|r} 
A13 & 2.01 & 2.09 & 0.50 & 0.22 & $2.28^{\mathrm{b}}$ \\
A14 & 2.32 & 2.55 & $-^{\mathrm{a}}$ & - & $2.28^{\mathrm{b}}$ \\
A15 & 2.77 & 2.81 & $-{ }^{\mathrm{a}}$ & 1.48 & $2.28^{\mathrm{b}}$ \\
A16 & 1.84 & 1.90 & 0.34 & 0.19 & $3.04^{\mathrm{b}}$ \\
A17 & 2.19 & 2.28 & 0.59 & 0.25 & $2 \times 1.39^{\mathrm{b}}$ \\
A18 & 1.49 & 1.65 & 0.29 & 0.18 & $4.00^{\mathrm{b}}$ \\
\hline
\end{tabular}

${ }^{a}$ Bands show no dispersion.

${ }^{\mathrm{b}}$ Values from ref. 52.

${ }^{\mathrm{c}}$ Calculated relative to benzene from ref. 53 . 


\section{DISCUSSION}

TD-DFT vs GW-BSE. It is well documented that hybrid DFT gives good predictions of $E_{g}$ for both molecular ${ }^{51,54-58}$ and polymer ${ }^{56,59}$ OSCs, as well as MOFs, ${ }^{60-63}$ which is partially due to the possibility of using different functionals with varying levels of Hartree-Fock exchange. Our HSE and TD-HSE calculations show that admixing 25\% HF exchange produces effective masses that are similar to GW and results in a reasonable agreement of predicted optical spectra with experiment. This strongly suggests that results obtained on related systems in sections (h), (i), and (j) are useful, although some caveats apply. First, correcting the DFTobtained $E_{\mathrm{g}}$ for ZGR (Figure 12) results in a much worse agreement with experiment. This is partially a consequence of the fact that hybrid TD-DFT results are most frequently directly compared to experimental $E_{\mathrm{g}}$ values, leading to an increase in popularity of functionals that produce good results "out of the box", such as B3LYP or HSE. ${ }^{54,57,58}$ Ignoring the effect of electron-phonon coupling on the band gap is certainly valid for inorganics with heavy atoms, but both our results and previous experimental and computational work find values around 0.2-0.3 eV for carbon-based compounds, ${ }^{64}$ highlighting the importance of including zero-gap renormalization in OSCs. This may be especially important in cases where a precise value of $E_{\mathrm{g}}$ is needed, such as in sensors or light-gathering devices.

The second issue with TD-HSE is its approximate treatment of charge screening. This results in a severe underestimation of $E_{\mathrm{xb}}$ compared to GW-BSE, which is known to show very good agreement with experiments. ${ }^{65}$ These results suggest that TD-HSE likely profits from fortuitous error cancellation (i.e., underestimation of both the electronic band gap and $E_{\mathrm{xb}}$ ), as well as from the fact that ZGR has not been extensively studied in OSCs. Therefore, the usefulness of TD-HSE for the estimation of precise values of $E_{\mathrm{g}}$ and $E_{\mathrm{xb}}$, which is important for determining applicability in devices such as LEDs (high $E_{\mathrm{xb}}$ ) or solar cells (low $E_{\mathrm{xb}}$ ) may be limited, and the use of GW-BSE(-ZGR) may be more appropriate.

On the other hand, while the result obtained by GW-BSE-ZGR is in excellent agreement with experiment, it is also partly a product of good fortune, as the GW calculation is only converged to about $0.05 \mathrm{eV}$ (see Figure S6), the vertex corrections are ignored, and there is a slight discrepancy between the experimental and optimized geometries. A more serious issue with GW calculations is their very poor scaling with regard to system size (although low-scaling approaches ${ }^{66-69}$ are emerging), which is a serious limitation when considering OSCs; however, our results suggest that even an approximate calculation might provide important insights over hybrid DFT. 


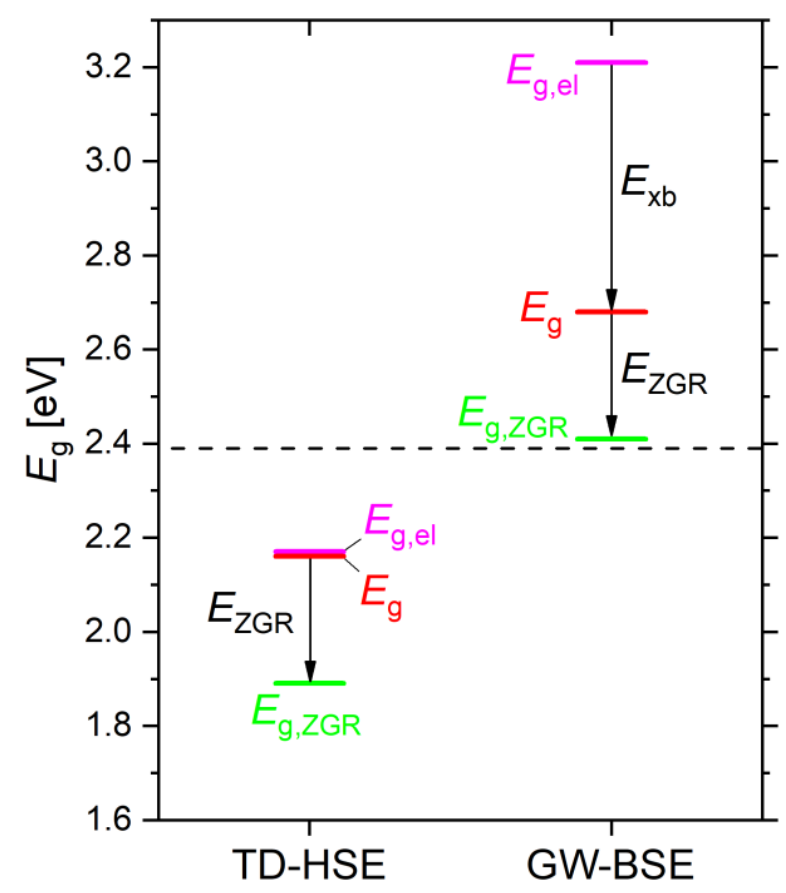

Figure 12. The band gap values of $\mathbf{C 1}$ obtained by TD-HSE and GW-BSE. The electronic band gap $\left(E_{\mathrm{g}, \mathrm{el}}\right)$ is shown in purple, the optical gap $\left(E_{\mathrm{g}}\right)$ in red, and the ZGRcorrected optical gap $\left(E_{\mathrm{g}, \mathrm{ZGR}}\right)$ in green. The experimental value of $E_{\mathrm{g}}$ is shown with a dashed black line.

The effect of solid-state packing can be estimated through its influence on the following: (i) the vibrational structure, (ii) the geometry, and (iii) the screening of Coulombic interaction. The effect of (i) is very minor (Figure 3), which is also illustrated by virtually identical ZGR values for $\mathbf{1}(-0.28 \mathrm{eV})$ and $\mathbf{C 1}(-0.27 \mathrm{eV})$, and similar phonon band structures (Figure 6). The influence of packing on the polymer geometry (ii) can mostly be described through changes in $\theta\left(37.5^{\circ}\right.$ in $\mathbf{1}$ vs $53.8^{\circ}$ in $\left.\mathbf{C 1}\right)$, which result in an $E_{\mathrm{g}}$ increase of $0.67 \mathrm{eV}$ at the GW-BSE level. The electron-hole interactions (iii) are screened much more strongly in $\mathbf{C 1}$, which significantly reduces $E_{\mathrm{xb}}(0.55 \mathrm{eV}$ for $\mathbf{C 1}$ vs $1.47 \mathrm{eV}$ for $\mathbf{1})$ of the first optical transition. Therefore, (i) is unimportant, while the effects of (ii) and (iii) are significant and opposite to each other (Figure 13). The geometry-independent effect of solid-state packing on $E_{\mathrm{g}}$, denoted as $E^{3 \mathrm{D}}$, can be estimated by comparing the $E_{\mathrm{g}}$ values of $\mathbf{C} \mathbf{1}$ and a $\mathbf{1}$ with the geometry (i.e. $\theta$ ) of $\mathbf{C 1}$, which produces a value of $0.65 \mathrm{eV}$ (Figure 13) at the GW-BSE-ZGR level.

A qualitatively similar result is obtained by DFT on the geometries modulated along normal modes which correspond to a rotation about $\theta$ (Figure 8). In that case, $E^{3 \mathrm{D}}$ can be estimated as $0.24 \mathrm{eV}$ from linear regression for $\mathbf{1}$.

The effect of solid-state packing on the transport properties is also significant. Although $\mathbf{C 1}$ has significantly larger effective masses than $\mathbf{1}$, its band curvatures in the $k_{\mathrm{x}}$ and $k_{\mathrm{z}}$ directions are similar. This translates to similar mobility along the polymer chain $(\mathrm{x})$ and the direction of $\pi-\pi$ stacking (z), suggesting that even polycrystalline films of $\mathbf{C 1}$ may show electrical conductivity with doping, which we will attempt to measure by four-point and contactless ${ }^{70}$ measurements in a forthcoming study. 


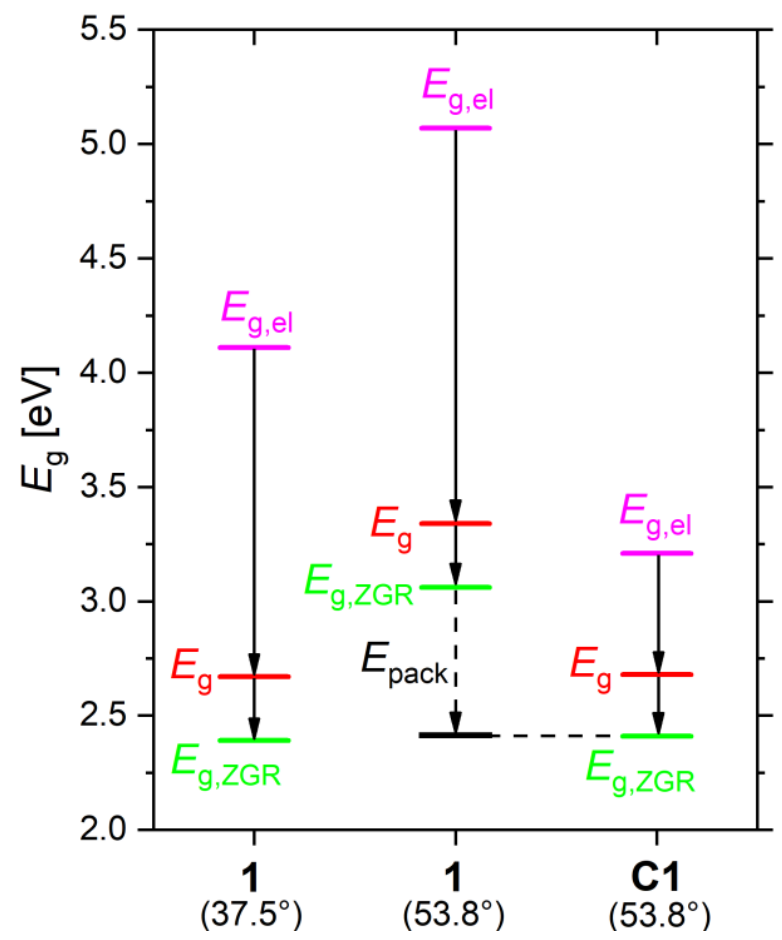

Figure 13. The electronic band gap $\left(E_{\mathrm{g}, \mathrm{el}}\right.$, purple) optical gap $\left(E_{\mathrm{g}}\right.$, red) and the ZGRcorrected optical gap ( $E_{\mathrm{g}, \mathrm{ZGR}}$, green) obtained using GW-BSE-ZGR for 1 with different $\theta$ values (in parentheses) and $\mathbf{C 1}$.

The effect of torsion presents itself as a linear dependence of $E_{\mathrm{g}}$ on $\theta$, as illustrated by DFT results in Figure 8. In the case of 1, GW-BSE results predict a larger slope $\left(0.041 \mathrm{eV} /{ }^{\circ}\right)$ than DFT $\left(0.024 \mathrm{eV} /{ }^{\circ}\right)$, which may be due to a better description of exciton (de)localization upon changes in $\theta$. DFT predicts a stronger $E_{\mathrm{g}}-\theta$ dependence (slope of $0.034 \mathrm{eV} /{ }^{\circ}$ ) for $\mathbf{C} \mathbf{1}$ than for $1\left(0.024 \mathrm{eV} /^{\circ}\right)$, which can be attributed to the effect through-space $\pi-\pi$ interactions between the polymer strands. These interactions also cause a relatively large $\left(16.3^{\circ}\right)$ change in $\theta$ of $\mathbf{C 1}$ relative to $\mathbf{1}$, showing that packing effects and torsion are coupled, but may be separated (Figure 13). Therefore, $E^{\theta}$ may be understood as a parameter causing a linear change of $E_{\mathrm{g}}$ with $\theta$, the magnitude of which can be determined computationally.

Changes in $E_{\mathrm{g}}$ occurring upon substitution (Figure 8) can mostly be attributed to a change in $\theta$. Also, estimating $E_{\mathrm{g}}$ of the $\mathbf{1} @ \mathbf{A u}$ monolayer by assuming that the changes occur only due to torsion (Table 3, method B) gives a very good estimate of the value determined by spectroscopic ellipsometry. Therefore, many changes occurring upon modification of $\mathbf{1}$ can be understood purely through their effects on $\theta$, illustrating that the control of torsion is highly important for tuning the properties of conjugated polymers such as $\mathbf{1}$.

Effect of chemical modification. In all cases, the addition of substituents to the phenyl ring (S2-S9) or introducing nitrogen in the aromatic core (A10-A12) results in band localization, which is reflected in a significant increase in effective masses (Table 4) and $E_{\mathrm{g}}$ (Figure 8). As mentioned in the previous section, this can mostly be explained by an increase in $\theta$ caused by steric hinderance between the substituent and the azodioxide group. The nitro group (S6) is an exception, being the only one below the trendline in Figure 8, which is likely due to a very 
strong electron-withdrawing resonance effect $(\sigma=0.78)$ stabilizing the excited state by moving the transition density from the $\mathrm{C}-\mathrm{N}$ bond to the nitro group (cf. Figure $7 \mathrm{e}$ with Figure 14).

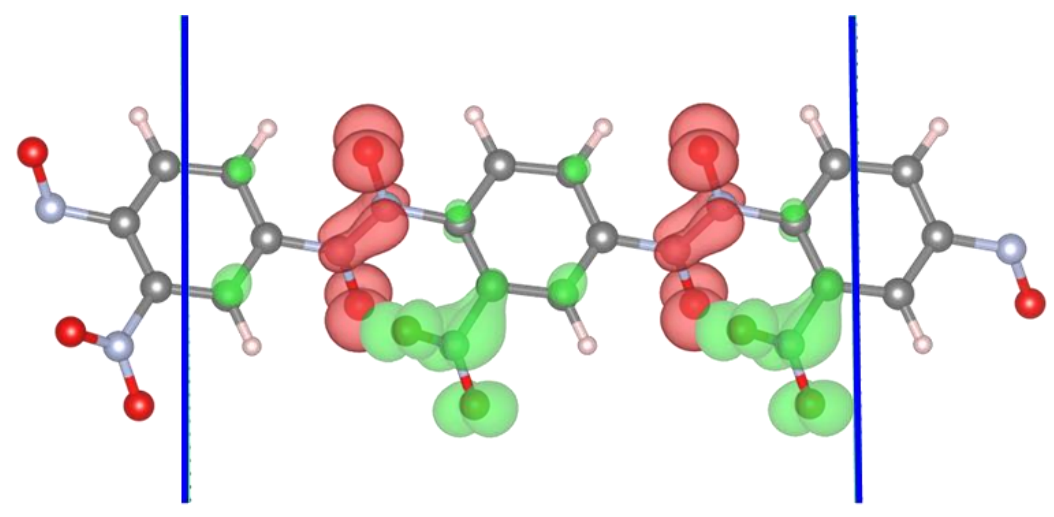

Figure 14. Transition density for the first optical transition in S6.

Replacing benzene with naphthalene (A13-A15) has more dramatic effects, which are highly dependent on the substituent position. The 2,6- substituted derivatives A13 and A16 show an increase in band curvature, while an almost complete flattening of the conducting and valence bands occurs in 2,7-substituted A14 and 1,5-substitued A15. The differences between these compounds can be rationalized by visualizing their orbitals or looking at the aromatic delocalization pathways (Figure 15), which suggest delocalization across multiple unit cells is present in A13, but absent in A14. Comparing 1 (benzene) with A13 (naphthalene), A16, (anthracene) and $\mathbf{A 1 8}$ (tetracene), we note that both $E_{\mathrm{g}}$ and effective masses decrease continuously with increasing conjugation. However, the differences between effective masses of A16 and A18 are minimal, suggesting that transport becomes limited by the azodioxide group. On the other hand, $E_{\mathrm{g}}$ continues to decrease significantly (1.84 eV in A16 vs $1.49 \mathrm{eV}$ in A18), presenting an interesting opportunity for band gap engineering by combining an appropriate $\left(E_{\mathrm{g}}\right.$-decreasing) aromatic core with an $\left(E_{\mathrm{g}}\right.$-increasing) substituent. This may be especially attractive for $\mathbf{A 1 8}$, as tetracene-based compounds are attractive candidates for singlet fission. ${ }^{71}$ Finally, the biphenyl-based A17 (which has been synthesized) ${ }^{42}$ shows a significant (30\%) enhancement of transport properties relative to $\mathbf{1}$.

(a)

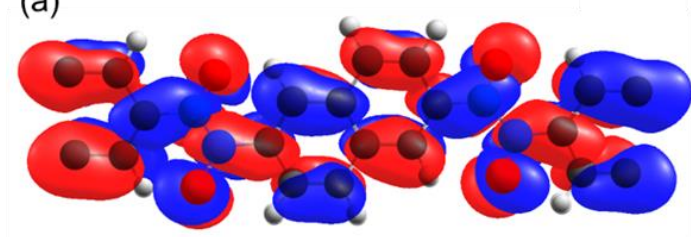

A13, $\mathrm{CB}$

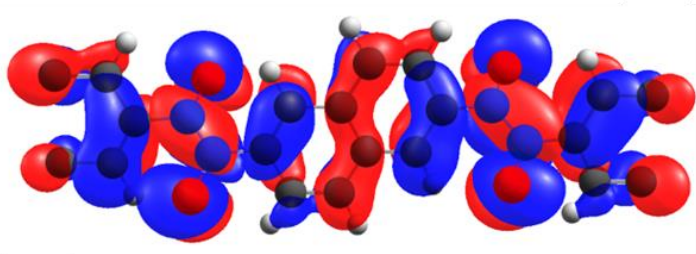

A13, VB
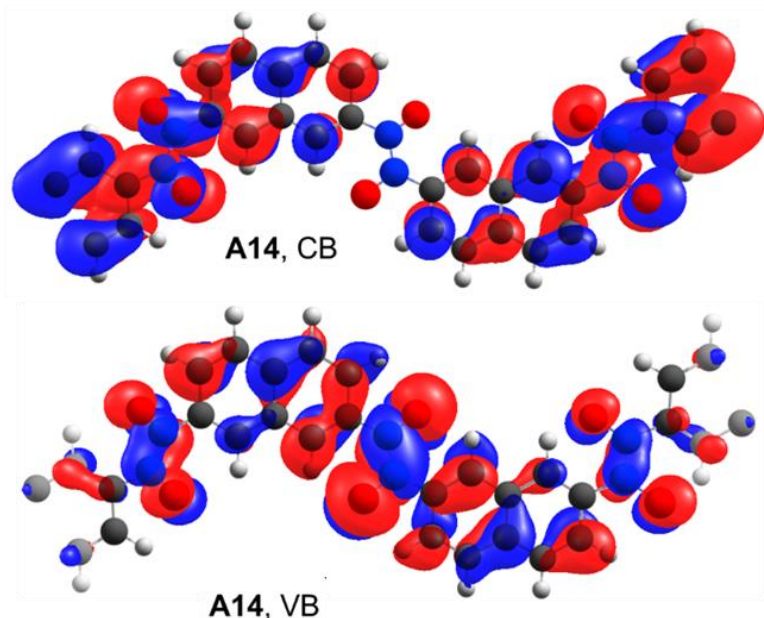

A14, VB 
(b)

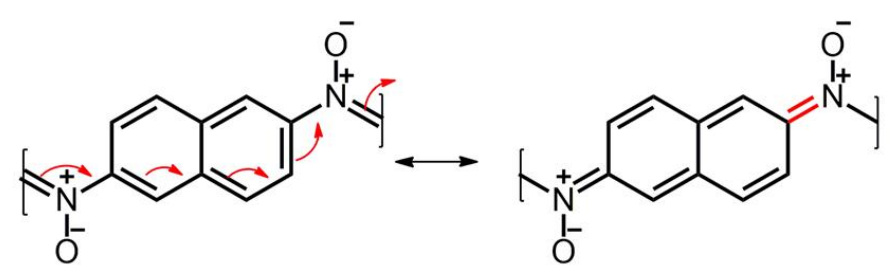

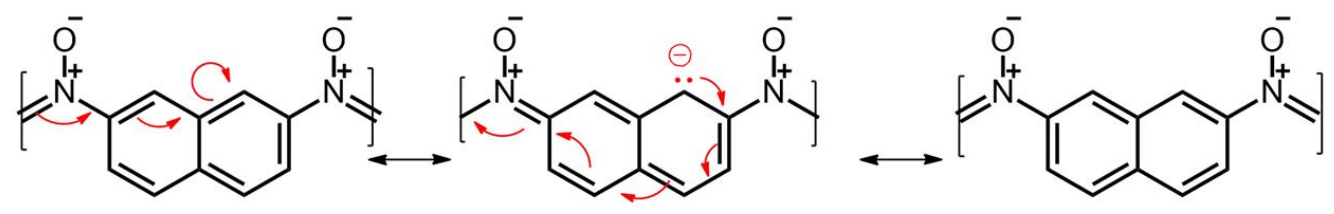

Figure 15. (a) Conduction (CB, top) and valence (VB, bottom) bands of A13 (top) and A14 (bottom) at $\Gamma$, and (b) possible resonance structures for A13 (top) and A14 (bottom).

Adsorption on gold. AFM reveals that the drop-casting method can be used to adsorb $\mathbf{1}$ on a (111) gold surface, producing thin films of 1 @ Au. Their morphology is characterized by islands with heights reaching 15 to $30 \mathrm{~nm}$, which is consistent with multilayer formation. Beneath the on-top islands, additional multilayers are likely to be present. This is further corroborated by ellipsometry, which estimates the average film thickness of $\mathbf{1} @ \mathbf{A u}$ at around $180 \mathrm{~nm}$. The presence of the IR peak $(\sim 1.0 \mathrm{eV})$ in the imaginary dielectric function of $\mathbf{1} @ \mathbf{A u}$ obtained by spectroscopic ellipsometry may be accounted for by calculations of the $\mathbf{1} @ \mathbf{A u}$ monolayer, which suggest that the $E_{\mathrm{g}}$ narrowing is caused by polymer planarization. In case of nitroso dimers, such planarization was already observed. ${ }^{30}$

Due to its interesting electronic properties and high stability, $\mathbf{1}$ could be possibly used as an organic field-effect transistor (OFET). However, for application in such devices, highly ordered OSC films are needed. ${ }^{72}$ To achieve this goal, a comprehensive investigation of the influence of experimental parameters (e.g. solution concentration, solvent, deposition method, substrate) on the surface morphology of thin films of $\mathbf{1}$, and its derivatives such as A16, A17, and A18, is planned. 


\section{CONCLUSION}

Experimental characterization of $\mathbf{C 1}$ demonstrates that it is a purely polymeric compound with an $E_{\mathrm{g}}$ of $2.39 \mathrm{eV}$. This value is very well reproduced by a GW-BSE calculation with ZGR corrections $(2.41 \mathrm{eV})$, demonstrating a nearly parameter-free procedure for calculating OSC properties. These calculations also predict that $\mathbf{C 1}$ has a relatively large exciton binding energy, which is strongly influenced by the environment. Crystal packing causes a significant increase of the torsional angle $\theta$ relative to a single strand of $\mathbf{1}$; however, this is nearly completely offset by the effect of 3D interactions. Significant band curvature along the polymer chain and $\pi-\pi$ stacking directions is found, suggesting 2-D conductivity may be present upon excitation or doping.

Hybrid TD-DFT predicts a linear dependence of $E_{\mathrm{g}}$ on torsion and suggests that substituent effects can mostly be understood through their effect on $\theta$. In contrast, extending the conjugation present in the aromatic core is predicted to enhance the transport properties and narrow $E_{\mathrm{g}}$, which may, in combination with adding substituents, allow for precise manipulation of the properties of derivatives of $\mathbf{C 1}$.

Finally, drop-casting is demonstrated as a viable method for producing thin films of $\mathbf{1}$ on a (111) gold surface, opening a possibility for preparing OFETs. Atomic force microscopy reveals these films display island-like morphology, while spectroscopic ellipsometry determines their band gap to be $0.68 \mathrm{eV}$. According to hybrid TD-DFT calculations, this large perturbation can mostly be attributed to surface-induced polymer planarization.

\section{EXPERIMENTAL PART}

Calculations. Functional choice. To identify a functional which produces a geometry in accordance with the experimentally determined structure of $\mathbf{C 1}$, we optimized its geometry using periodic boundary condition DFT calculations with several popular functionals in combination with different schemes to account for van der Waals interactions (Figure S1). The smallest RMSD values and lowest deviations in $\theta$ were obtained using the PBE-revD3BJ method, which combines the $\mathrm{PBE}^{73}$ functional with the corrected D3BJ correction scheme. ${ }^{46,74}$ This method was used in optimizations of $\mathbf{C 1}$ and all 1-D systems. All calculations except for the ZGR used a primitive unit cell (two dinitroso units for $\mathbf{C 1}$ and one unit for 1-D systems); unconstrained optimizations of supercells produced no significant desymmetrization. For modelling gold surface adsorption, the vdW-DF2 scheme was used, as it was shown to describe organic-metal interfaces well. ${ }^{75-78}$

Optimization. Geometries were optimized with the above described PBE-revD3BJ method. A $700 \mathrm{eV}$ kinetic energy cutoff for the planewave basis was used, and a $0.15 \mathrm{eV}$ Gaussian smearing was employed. Calculations on 1-D systems utilized a $\Gamma$-centered reciprocal grid with 16 k-points along the $\Gamma$-X path. The structure of $\mathbf{C 1}$ was optimized using an $8 \times 4 \times 12$ reciprocal grid. Geometry optimizations were carried out until the sum of Helmann-Feynman forces was less than $0.01 \mathrm{eV} \AA^{-1}$, while the wavefunction energy was converged to $10^{-6} \mathrm{eV} .{ }^{79}$

HSE and TD-HSE calculations. The HSE wavefunction was converged to $10^{-6} \mathrm{eV}$. Out of 324 bands (25 and 50 occupied for 1-D and 3D systems, respectively) that were used to construct the frequency-dependent dielectric matrix, 12 occupied and 36 virtual bands were included in the Casida equation. Band structures interpolation and calculation of transport properties were done with the BoltZtraP2 code. ${ }^{80}$ Band paths were drawn according to Hinuma et al. ${ }^{81}$ 
GW calculations were done on a PBE wavefunction with 502 bands, Gaussian smearing of $0.175 \mathrm{eV}$, and a $120 \mathrm{eV}$ response function cutoff. For $\mathbf{C 1}$, an $8 \times 1 \times 6$ Gamma-centered k-grid was used, while calculations on $\mathbf{1}$ used an equivalent $8 \times 1 \times 1$ grid. Four iterations were performed, which resulted in convergence within $<25 \mathrm{meV}$ in quasiparticle gaps. The TammDancoff approximation was used. Convergence tests can be found in Figure S6 in SI.

Zero-gap renormalization. The phonon-induced band-gap renormalization was estimated using the one-shot ZG configuration method. ${ }^{43,82}$ A $4 \times 2 \times 3$ supercell was used, with benchmarking details in Table S1 in SI.

All calculations were performed using the Vienna Ab initio Simulations Package (VASP). ${ }^{83-}$ 86

Synthesis. Compound 1 was prepared by the method described recently. ${ }^{29}$ The product was purified by column chromatography (silica gel, chloroform:acetone $=4: 1$ ) and the green solution of monomer of $\mathbf{1}$ was collected. Evaporation of the solvent yielded a yellow powder of $E$-azodioxy polymer 1 which was confirmed by IR spectroscopy.

Diffuse Reflectance Spectroscopy. The UV/Vis diffuse reflectance spectrum of a powder sample of $\mathbf{C 1}$ was obtained using the Cary 5000 (Agilent Technologies) integrating sphere spectrophotometer. Diffuse reflectance was modelled according to the Kubelka-Munk model, ${ }^{87}$ described as:

$$
\frac{K}{S}=\frac{\left(1-R_{\mathrm{inf}}\right)^{2}}{2 R_{\mathrm{inf}}} F\left(R_{\mathrm{inf}}\right)
$$

where $K$ and $S$ are phenomenological parameters and $F\left(R_{\text {inf }}\right)$ is the Kubelka-Munk reemission function. The absolute reflectance can be written as:

$$
R_{\text {inf }}=\frac{R_{\text {sample }}}{R_{\text {standard }}}
$$

where $R_{\text {standard }}=1$. For perfect diffuse scattering $K$ is equal to $2 \alpha$, where $\alpha$ is the absorption coefficient, with the energy dependence:

$$
\alpha E=C_{1}\left(E-E_{\mathrm{g}}\right)^{n}
$$

where $C_{1}$ is a proportionality constant, and $n$ is 0.5 for direct band gaps. As $S$ is a constant too, in the case of a direct band gap we can write:

$$
\left(F\left(R_{\mathrm{inf}}\right) E\right)^{2}=\left(\frac{2 \alpha}{S} E\right)^{2}=\left(\frac{2 C_{1}\left(E-E_{\mathrm{g}}\right)^{0,5}}{S E} E\right)^{2}=C_{2}\left(E-E_{\mathrm{g}}\right)
$$

which is equation (1) from which the $E_{\mathrm{g}}$ value of $\mathbf{C 1}$ was determined.

Infrared spectroscopy. The infrared spectrum of $\mathbf{C 1}$ was measured on a polycrystalline sample, while a spectrum of 1 was obtained by preparing a well-mixed 1:100 $\mathrm{KBr}$ matrix of C1. Spectra were recorded using a PerkinElmer SpectrumTwo spectrometer at a resolution of $4 \mathrm{~cm}^{-1}$, averaging 10 scans per spectrum.

Preparation of 1@Au. All glassware was cleaned using a piranha solution (3:1 mixture of sulfuric acid and $30 \%$ hydrogen peroxide heated to $90{ }^{\circ} \mathrm{C}$ ). Prior to deposition, commercially available $\mathrm{Au}(111) / \mathrm{mica}$ substrates (Phasis) were flame annealing followed by cooling in the stream of argon. Thin films of $\mathbf{1}$ (1@Au) were prepared by drop-casting method as follows: two droplets of $0.02 \mathrm{M}$ solution of compound 1 in a 4:1 mixture of chloroform and acetone 
were placed on an $\mathrm{Au}(111)$ surface. After the solvent evaporated, the adlayers were characterized by atomic force microscopy (AFM) and ellipsometry.

Atomic force microscopy (AFM). AFM measurements were performed on a MultiMode 8 (Bruker) in soft tapping mode with silicon probes (Bruker, NCHV-A, nom. spring constant $40 \mathrm{~N} / \mathrm{m}$, nom. freq. $320 \mathrm{kHz}$ ) under ambient conditions and humidity of 40-50\%. AFM images were processed and analyzed by using the NanoScope Analysis 2.0 (Bruker) software.

Ellipsometry. Optical characteristics of 1 deposited on a (111) gold surface were investigated by spectroscopic ellipsometry using a J. A. Woollam V-VASE ellipsometer in the spectral range from 0.57 to $4.73 \mathrm{eV}$. Measurements were carried out at three angles of incidence, $55^{\circ}, 65^{\circ}$ and $75^{\circ}$. Optical properties and layer thickness were both obtained by simultaneous fitting. Depolarization (due to thickness non-uniformity) was included into the model in order to ensure a satisfactory agreement between the experimental results and the data generated by the model. Due to complexity of the surface layer, the flexible multiple oscillator model was used. Due to the importance of estimating the band gap, Tauc-Lorentz oscillators were used in the general oscillator model because they include the band gap energy $\left(E_{\mathrm{g}}\right)$ as one of the defining parameters. ${ }^{88}$

\section{ASSOCIATED CONTENT}

Supporting Information. The Supporting Information is available free of charge on the ACS publication website.

Optimized geometries of all structures (POSCAR)

Benchmarking results for geometry optimization, GW calculations, and ZGR calculations.

Calculated conductivity of $\mathbf{C 1}$, full phonon band structure of $\mathbf{C 1}$, TD-HSE predicted optical spectra, Hammett graph for dinitroso dimers and polymers.

\section{AUTHOR INFORMATION}

\section{Corresponding Authors}

*ibiljan@chem.pmf.hr

*igor.roncevic@uochb.cas.cz

\section{ORCID}

I. Rončević: 0000-0003-2175-8059

I. Biljan: 0000-0002-0650-1063

\section{Notes}

The authors declare no competing financial interest.

\section{ACKNOWLEDGEMENTS}

Computational resources were supplied by the project "e-Infrastruktura CZ" (e-INFRA LM2018140) provided within the program Projects of Large Research, Development and Innovations Infrastructures. This work was supported by the Ministry of Education, Youth and Sports of the Czech Republic through the e-INFRA CZ (ID:90140). This work has been supported in part by Croatian Science Foundation under the project IP-2020-02-4467. The support of project CIuK co-financed by the Croatian Government and the European Union through the European Regional Development Fund - Competitiveness and Cohesion Operational Programme (Grant KK.01.1.1.02.0016) is acknowledged. We thank Professor Josef Michl (University of Boulder Colorado, USA) for valuable comments. 


\section{REFERENCES}

1. Wang, C., Dong, H., Hu, W., Liu, Y. \& Zhu, D. Semiconducting $\pi$-Conjugated Systems in Field-Effect Transistors: A Material Odyssey of Organic Electronics. Chemical Reviews 112, 2208-2267 (2011).

2. Dong, H., Wang, C. \& Hu, W. High performance organic semiconductors for fieldeffect transistors. Chemical Communications 46, 5211-5222 (2010).

3. Mei, J., Diao, Y., Appleton, A. L., Fang, L. \& Bao, Z. Integrated Materials Design of Organic Semiconductors for Field-Effect Transistors. Journal of the American Chemical Society 135, 6724-6746 (2013).

4. Yamashita, Y. Organic semiconductors for organic field-effect transistors. http://www.tandfonline.com/action/journalInformation? show=aimsScope\&journalCod $e=$ tsta20\#.VmBmuzZFCUk 10, 9 (2009).

5. Lin, Y., Li, Y. \& Zhan, X. Small molecule semiconductors for high-efficiency organic photovoltaics. Chemical Society Reviews 41, 4245-4272 (2012).

6. Facchetti, A. Polymer donor-polymer acceptor (all-polymer) solar cells. Materials Today 16, 123-132 (2013).

7. Dou, L., Liu, Y., Hong, Z., Li, G. \& Yang, Y. Low-Bandgap Near-IR Conjugated Polymers/Molecules for Organic Electronics. Chemical Reviews 115, 12633-12665 (2015).

8. Myers, J. D. \& Xue, J. Organic Semiconductors and their Applications in Photovoltaic Devices. http://dx.doi.org/10.1080/15583724.2011.644368 52, 1-37 (2012).

9. Li, C. \& Shi, G. Polythiophene-Based Optical Sensors for Small Molecules. ACS Applied Materials and Interfaces 5, 4503-4510 (2013).

10. Mako, T. L., Racicot, J. M. \& Levine, M. Supramolecular Luminescent Sensors. Chemical Reviews 119, 322-477 (2018).

11. Borges-González, J., Kousseff, C. J. \& Nielsen, C. B. Organic semiconductors for biological sensing. Journal of Materials Chemistry C 7, 1111-1130 (2019).

12. Liu, Y., He, K., Chen, G., Leow, W. R. \& Chen, X. Nature-Inspired Structural Materials for Flexible Electronic Devices. Chemical Reviews 117, 12893-12941 (2017).

13. Park, S., Loke, G., Fink, Y. \& Anikeeva, P. Flexible fiber-based optoelectronics for neural interfaces. Chemical Society Reviews 48, 1826-1852 (2019).

14. Wang, Y. et al. Organic crystalline materials in flexible electronics. Chemical Society Reviews 48, 1492-1530 (2019).

15. Bronstein, H., Nielsen, C. B., Schroeder, B. C. \& McCulloch, I. The role of chemical design in the performance of organic semiconductors. Nature Reviews Chemistry 2020 4:2 4, 66-77 (2020). 
16. Posligua, V. et al. Band Structures of Periodic Porphyrin Nanostructures. The Journal of Physical Chemistry C 122, 23790-23798 (2018).

17. Kato, T. Dependence of electron-phonon interactions on doped carriers in the negatively fractionally charged polyacene molecular crystals. Journal of Physical Chemistry C 115, 21383-21389 (2011).

18. Bettinger, H. F., Tönshoff, C., Doerr, M. \& Sanchez-Garcia, E. Electronically Excited States of Higher Acenes up to Nonacene: A Density Functional Theory/Multireference Configuration Interaction Study. Journal of Chemical Theory and Computation 12, 305-312 (2016).

19. Bursi, L., Calzolari, A., Corni, S. \& Molinari, E. Light-Induced Field Enhancement in Nanoscale Systems from First-Principles: The Case of Polyacenes. ACS Photonics 1, 1049-1058 (2014).

20. Kaloni, T. P., Schreckenbach, G. \& Freund, M. S. Band gap modulation in polythiophene and polypyrrole-based systems. Scientific Reports 2016 6:1 6, 1-18 (2016).

21. Jayasundara, W. J. M. J. S. R. \& Schreckenbach, G. Theoretical Study of p-and nDoping of Polythiophene-and Polypyrrole-Based Conjugated Polymers. Journal of Physical Chemistry C 124, 17528-17537 (2020).

22. Reining, L. The GW approximation: content, successes and limitations. Wiley Interdisciplinary Reviews: Computational Molecular Science 8, e1344 (2018).

23. Golze, D., Dvorak, M. \& Rinke, P. The GW compendium: A practical guide to theoretical photoemission spectroscopy. Frontiers in Chemistry 7, 377 (2019).

24. Leng, X., Jin, F., Wei, M. \& Ma, Y. GW method and Bethe-Salpeter equation for calculating electronic excitations. Wiley Interdisciplinary Reviews: Computational Molecular Science 6, 532-550 (2016).

25. Alves-Santos, M., Jorge, L. M. M., Caldas, M. J. \& Varsano, D. Electronic Structure of Interfaces between Thiophene and TiO2 Nanostructures. Journal of Physical Chemistry C 118, 13539-13544 (2014).

26. Atambo, M. O. et al. Electronic and optical properties of doped $\mathrm{TiO} 2$ by many-body perturbation theory. Physical Review Materials 3, 045401 (2019).

27. Ahmed, T., Albers, R. C., Balatsky, A. v., Friedrich, C. \& Zhu, J. X. G W quasiparticle calculations with spin-orbit coupling for the light actinides. Physical Review B Condensed Matter and Materials Physics 89, 035104 (2014).

28. Radha, S. K. et al. Optical response and band structure of $\mathrm{LiCoO} 2$ including electronhole interaction effects. Physical Review B 104, 115120 (2021).

29. Gallo, G., Mihanović, A., Rončević, I., Dinnebier, R. \& Vančik, H. Crystal structure and ON-OFF polymerization mechanism of poly(1,4-phenyleneazine-N,N-dioxide), a possible wide bandgap semiconductor. Polymer 214, 123235 (2021). 
30. Vančik, H. Aromatic C-nitroso compounds. Aromatic C-Nitroso Compounds 9789400763371, 1-156 (2013).

31. Beaudoin, D. \& Wuest, J. D. Dimerization of Aromatic C-Nitroso Compounds. Chemical Reviews 116, 258-286 (2016).

32. Vančik, H. et al. Solid state photochromism and thermochromism in nitroso monomerdimer equilibrium. Journal of Physical Chemistry B 106, 1576-1580 (2002).

33. Halasz, I., Meštrović, E., Čičak, H., Mihalić, Z. \& Vančik, H. Solid-State Reaction Mechanisms in Monomer-Dimer Interconversions of p- Bromonitrosobenzene. SingleCrystal-to- Single-Crystal Photodissociation and Formation of New Non-van der Waals Close Contact. Journal of Organic Chemistry 70, 8461-8467 (2005).

34. Varga, K. et al. Thermally-induced reactions of aromatic crystalline nitroso compounds. Chemistry Select 4, 4709-4717 (2019).

35. Okamoto, T. et al. Robust, high-performance n-type organic semiconductors. Science Advances 6, (2020).

36. Brédas, J. L., Calbert, J. P., da Silva Filho, D. A. \& Cornil, J. Organic semiconductors: A theoretical characterization of the basic parameters governing charge transport. Proceedings of the National Academy of Sciences 99, 5804-5809 (2002).

37. Beaudoin, D., Maris, T. \& Wuest, J. D. Constructing monocrystalline covalent organic networks by polymerization. Nature chemistry 5, 830-834 (2013).

38. Roncali, J. Molecular Engineering of the Band Gap of $\pi$-Conjugated Systems: Facing Technological Applications. Macromolecular Rapid Communications 28, 1761-1775 (2007).

39. Huang, H., Yang, L., Facchetti, A. \& Marks, T. J. Organic and Polymeric Semiconductors Enhanced by Noncovalent Conformational Locks. Chemical Reviews 117, 10291-10318 (2017).

40. Krukau, A. v., Vydrov, O. A., Izmaylov, A. F. \& Scuseria, G. E. Influence of the exchange screening parameter on the performance of screened hybrid functionals. The Journal of Chemical Physics 125, 224106 (2006).

41. Gowenlock, B. G. \& Richter-Addo, G. B. Dinitroso and polynitroso compounds. Chemical Society Reviews 34, 797-809 (2005).

42. Vancik, H., Roncevic, I., Bibulic, P., Spadina, M. \& Biljan, I. Isothermal and Isoconversional Modeling of Solid-State Nitroso Polymerization. The journal of physical chemistry. A, Molecules, spectroscopy, kinetics, environment, \& general theory 124, 10726-10735 (2020).

43. Karsai, F., Engel, M., Kresse, G. \& Flage-Larsen, E. Electron-phonon coupling in semiconductors within the GW approximation. New Journal of Physics 20, 123008 (2018).

44. Miglio, A. et al. Predominance of non-adiabatic effects in zero-point renormalization of the electronic band gap. npj Computational Materials 2020 6:1 6, 1-8 (2020). 
45. Alvertis, A. M. et al. Impact of exciton delocalization on exciton-vibration interactions in organic semiconductors. Physical Review B 102, (2020).

46. Smith, D. G. A., Burns, L. A., Patkowski, K. \& Sherrill, C. D. Revised Damping Parameters for the D3 Dispersion Correction to Density Functional Theory. Journal of Physical Chemistry Letters 7, 2197-2203 (2016).

47. Bibulić, P., Rončević, I., Varga, K., Mihalić, Z. \& Vančik, H. Structure and topochemistry of azodioxide oligomers in solid state. Journal of molecular structure 1104, 85-90 (2016).

48. Hacker, N. P. Investigation of the polymerization of 1,4-dinitrosobenzene by lowtemperature infrared and UV absorption spectroscopy. Macromolecules 26, 5937-5942 (1993).

49. Shishkin, M. \& Kresse, G. Self-consistent GW calculations for semiconductors and insulators. Physical Review B - Condensed Matter and Materials Physics 75, 235102 (2007).

50. Lee, K., Murray, É. D., Kong, L., Lundqvist, B. I. \& Langreth, D. C. Higher-accuracy van der Waals density functional. Physical Review B - Condensed Matter and Materials Physics 82, 081101 (2010).

51. Kim, S. Y., Kim, M. J., Ahn, M., Lee, K. M. \& Wee, K. R. Systematic energy band gap control of pyrene based donor-acceptor-donor molecules for efficient chemosensor. Dyes and Pigments 191, 109362 (2021).

52. George, P. Critique of the resonance energy concept with particular reference to nitrogen heterocycles, especially porphyrins. Chemical Reviews 75, 85-111 (1975).

53. Maria, Nisa, R. U., Hanif, M., Mahmood, A. \& Ayub, K. Aromaticities of azines relative to benzene; a theoretical approach through the dimethyldihydropyrene probe. Journal of Physical Organic Chemistry 27, 860-866 (2014).

54. Privitera, A., Londi, G., Riede, M., D’Avino, G. \& Beljonne, D. Molecular Quadrupole Moments Promote Ground-State Charge Generation in Doped Organic Semiconductors. Advanced Functional Materials 30, (2020).

55. Méndez, H. et al. Doping of organic semiconductors: Impact of dopant strength and electronic coupling. Angewandte Chemie - International Edition 52, 7751-7755 (2013).

56. Körzdörfer, T. \& Brédas, J. L. Organic electronic materials: Recent advances in the dft description of the ground and excited states using tuned range-separated hybrid functionals. Accounts of Chemical Research 47, 3284-3291 (2014).

57. Panidi, J. et al. Remarkable Enhancement of the Hole Mobility in Several Organic Small-Molecules, Polymers, and Small-Molecule:Polymer Blend Transistors by Simple Admixing of the Lewis Acid p-Dopant B(C6F5)3. Advanced Science 5, (2018). 
58. Welch, G. C., Coffin, R., Peet, J. \& Bazan, G. C. Band gap control in conjugated oligomers via Lewis acids. Journal of the American Chemical Society 131, 1080210803 (2009).

59. Marqués, P. S. et al. Understanding how Lewis acids dope organic semiconductors: a “complex" story. Chemical Science 12, 7012-7022 (2021).

60. Sarkisov, L. \& Kim, J. Computational structure characterization tools for the era of material informatics. Chemical Engineering Science 121, 322-330 (2015).

61. Coudert, F. X. \& Fuchs, A. H. Computational characterization and prediction of metalorganic framework properties. Coordination Chemistry Reviews 307, 211-236 (2016).

62. Odoh, S. O., Cramer, C. J., Truhlar, D. G. \& Gagliardi, L. Quantum-Chemical Characterization of the Properties and Reactivities of Metal-Organic Frameworks. Chemical Reviews 115, 6051-6111 (2015).

63. Wilbraham, L., Coudert, F. X. \& Ciofini, I. Modelling photophysical properties of metal-organic frameworks: a density functional theory based approach. Physical Chemistry Chemical Physics 18, 25176-25182 (2016).

64. Miglio, A. et al. Predominance of non-adiabatic effects in zero-point renormalization of the electronic band gap. npj Computational Materials 2020 6:1 6, 1-8 (2020).

65. Kolos, M. \& Karlický, F. Accurate many-body calculation of electronic and optical band gap of bulk hexagonal boron nitride. Physical Chemistry Chemical Physics 21, 3999-4005 (2019).

66. Förster, A. \& Visscher, L. Low-Order Scaling Quasiparticle Self-Consistent GW for Molecules. Frontiers in Chemistry 9, (2021).

67. del Ben, M. et al. Large-scale GW calculations on pre-exascale HPC systems. Computer Physics Communications 235, 187-195 (2019).

68. Liu, P., Kaltak, M., Klimeš, J. \& Kresse, G. Cubic scaling GW: Towards fast quasiparticle calculations. Physical Review B 94, 165109 (2016).

69. Wilhelm, J., Golze, D., Talirz, L., Hutter, J. \& Pignedoli, C. A. Toward GW Calculations on Thousands of Atoms. Journal of Physical Chemistry Letters 9, 306312 (2018).

70. Ferguson, A. J., Kopidakis, N., Shaheen, S. E. \& Rumbles, G. Dark Carriers, Trapping, and Activation Control of Carrier Recombination in Neat P3HT and P3HT:PCBM Blends. Journal of Physical Chemistry C 115, 23134-23148 (2011).

71. Aguilar Suarez, L. E., Menger, M. F. S. J. \& Faraji, S. Singlet fission in tetracene: an excited state analysis. https://doi.org/10.1080/00268976.2020.1769870 118, 21-22 (2020).

72. Izawa, S. et al. Crystallization and Polymorphism of Organic Semiconductor in Thin Film Induced by Surface Segregated Monolayers. Scientific Reports 2017 8:1 8, 1-11 (2018). 
73. Perdew, J. P., Burke, K. \& Ernzerhof, M. Generalized Gradient Approximation Made Simple. Physical Review Letters 77, 3865 (1996).

74. Grimme, S., Antony, J., Ehrlich, S. \& Krieg, H. A consistent and accurate ab initio parametrization of density functional dispersion correction (DFT-D) for the 94 elements H-Pu. The Journal of Chemical Physics 132, 154104 (2010).

75. Carrasco, J., Liu, W., Michaelides, A. \& Tkatchenko, A. Insight into the description of van der Waals forces for benzene adsorption on transition metal (111) surfaces.

Citation: The Journal of Chemical Physics 140, 84704 (2014).

76. Yuan, D., Liao, H. \& Hu, W. Assessment of van der Waals inclusive density functional theory methods for adsorption and selective dehydrogenation of formic acid on Pt(111) surface. Physical Chemistry Chemical Physics 21, 21049-21056 (2019).

77. Vlaisavljevich, B. et al. Performance of van der Waals Corrected Functionals for Guest Adsorption in the M2(dobdc) Metal-Organic Frameworks. Journal of Physical Chemistry A 121, 4139-4151 (2017).

78. Tillotson, M., Brett, P., Bennett, R. A. \& Grau-Crespo, R. Adsorption of organic molecules at the TiO2(110) surface: the effect of van der Waals interactions. Surface Science 632, 142-153 (2014).

79. Togo, A. \& Tanaka, I. First principles phonon calculations in materials science. Scripta Materialia 108, 1-5 (2015).

80. Madsen, G. K. H., Carrete, J. \& Verstraete, M. J. BoltzTraP2, a program for interpolating band structures and calculating semi-classical transport coefficients. Computer Physics Communications 231, 140-145 (2018).

81. Hinuma, Y., Pizzi, G., Kumagai, Y., Oba, F. \& Tanaka, I. Band structure diagram paths based on crystallography. Computational Materials Science 128, 140-184 (2017).

82. Zacharias, M. \& Giustino, F. One-shot calculation of temperature-dependent optical spectra and phonon-induced band-gap renormalization. Physical Review B 94, 075125 (2016).

83. Kresse, G. \& Hafner, J. Ab initio molecular dynamics for liquid metals. Physical Review B 47, 558 (1993).

84. Kresse, G. \& Hafner, J. Ab initio molecular-dynamics simulation of the liquid-metalamorphous-semiconductor transition in germanium. Physical Review B 49, 14251 (1994).

85. Kresse, G. \& Furthmüller, J. Efficiency of ab-initio total energy calculations for metals and semiconductors using a plane-wave basis set. Computational Materials Science $\mathbf{6}$, 15-50 (1996).

86. Kresse, G. \& Furthmüller, J. Efficient iterative schemes for ab initio total-energy calculations using a plane-wave basis set. Physical Review B 54, 11169 (1996). 
87. Kubelka P \& Munk F. Ein Beitrag Zur Optik Der Farbanstriche. Z. Techn. Phys. 593601

https://www.scirp.org/(S(i43dyn45teexjx455qlt3d2q))/reference/ReferencesPapers.asp $\mathrm{x}$ ?ReferenceID=16426 (1931).

88. Jellison, G. E. \& Modine, F. A. Parameterization of the optical functions of amorphous materials in the interband region. Applied Physics Letters 69, 371 (1998). 